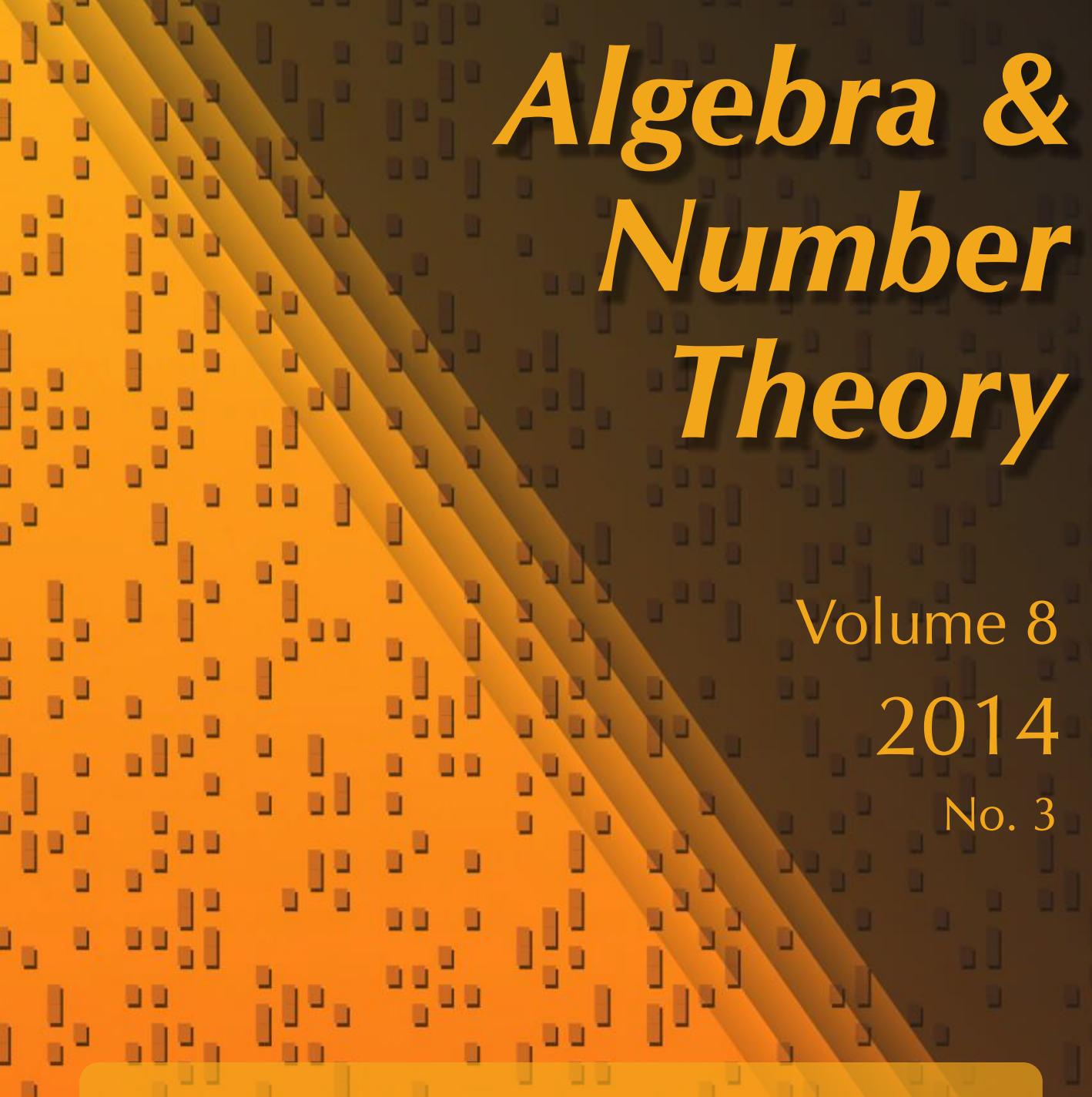

The tame-wild principle for discriminant relations for number fields John W. Jones and David P. Roberts

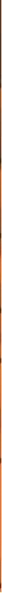




\title{
The tame-wild principle for discriminant relations for number fields
}

\author{
John W. Jones and David P. Roberts
}

\begin{abstract}
Consider tuples $\left(K_{1}, \ldots, K_{r}\right)$ of separable algebras over a common local or global number field $F$, with the $K_{i}$ related to each other by specified resolvent constructions. Under the assumption that all ramification is tame, simple group-theoretic calculations give best possible divisibility relations among the discriminants of $K_{i} / F$. We show that for many resolvent constructions, these divisibility relations continue to hold even in the presence of wild ramification.
\end{abstract}

\section{Overview}

Let $G$ be a finite group and let $\phi_{1}, \ldots, \phi_{r}$ be permutation characters of $G$. We say that a tuple $\left(K_{1}, \ldots, K_{r}\right)$ of separable algebras over a common ground field $F$ has type $\left(G, \phi_{1}, \ldots, \phi_{r}\right)$ if for a joint splitting field $K^{\mathrm{gal}}$ one can identify $\mathrm{Gal}\left(K^{\mathrm{gal}} / F\right)$ with a subgroup of $G$ such that the action of $\operatorname{Gal}\left(K^{\mathrm{gal}} / F\right)$ on $\operatorname{Hom}_{F}\left(K_{i}, K^{\mathrm{gal}}\right)$ has character $\phi_{i}$.

When $F$ is a local or global number field, one has discriminants $\mathfrak{D}_{K_{i} / F}$ which are ideals in the ring of integers $\mathcal{O}_{F}$ of $F$. One can ask for the strongest divisibility relations among these discriminants which hold as $\left(K_{1}, \ldots, K_{r}\right) / F$ varies over all possibilities of a given type $\left(G, \phi_{1}, \ldots, \phi_{r}\right)$. This question has a simple grouptheoretic answer if one restricts attention to tuples for which all ramification in each $K_{i} / F$ is tame.

This paper focuses on the following phenomenon: for many $\left(G, \phi_{1}, \ldots, \phi_{r}\right)$, the divisibility relations for tame $\left(K_{1}, \ldots, K_{r}\right) / F$ of type $\left(G, \phi_{1}, \ldots, \phi_{r}\right)$ hold also for arbitrary $\left(K_{1}, \ldots, K_{r}\right) / F$ of type $\left(G, \phi_{1}, \ldots, \phi_{r}\right)$. In this case, we say that the tame-wild principle holds for $\left(G, \phi_{1}, \ldots, \phi_{r}\right)$. Our terminology "tame-wild principle" is intended to be reminiscent of the standard terminology "local-global principle": we are showing in this paper that simple tame computations can often solve a complicated wild problem, just as simple local computations can often solve a complicated global problem.

This work was partially supported by a grant from the Simons Foundation (\#209472 to David Roberts). MSC2010: primary 11S15; secondary 11S20, 11R32.

Keywords: number field, discriminant, ramification. 
Section 2 provides an introductory example. Section 3 reviews some ramification theory centering on Artin characters, placing it in a framework which will be convenient for us. Section 4 states the tame-wild principle and gives two simple methods for proving instances of it.

If the tame-wild principle holds for a fixed $G$ and any $\left(\phi_{1}, \ldots, \phi_{r}\right)$ then we say it holds universally for $G$. Section 5 proves that the tame-wild principle holds universally for $G$ in a small class of groups we call U-groups. Section 6 considers the remaining groups, called $\mathrm{N}$-groups, finding that the tame-wild principle usually does not hold universally for them.

Sections 7 and 8 return to the more practical situation where one is given not only $G$ but also a small list of naturally arising $\phi_{i}$. Our theme is that the tame-wild principle is likely to hold, despite the negative results on N-groups. Section 7 focuses on comparing an arbitrary algebra $K / F$ with its splitting field $K^{\mathrm{gal}} / F$, proving that one of the two divisibility statements coming from the tame-wild principle holds for arbitrary $G$. Section 8 gives a collection of examples exploring the range of $\left(G, \phi_{1}, \ldots, \phi_{r}\right)$ for which the tame-wild principle holds.

This paper is written with applications to tabulating number fields of small discriminant in mind. The topics in Section 2B, Section 7E, and Section 8A all relate to this application. Moreover, as we will make clear, the theory we present here still applies when permutation characters $\phi_{i}$ are replaced by general characters $\chi_{i}$, and discriminants are correspondingly generalized to conductors. Applications to Artin $L$-functions of small conductor will be presented elsewhere.

\section{An introductory example}

In Section 2A, we provide an introductory instance of the tame-wild principle that we will revisit later to provide simple illustrations of general points. In Section 2B, we illustrate how this instance of the tame-wild principle gives an indirect but efficient way of solving a standard problem in tabulating number fields.

\section{A. The tame-wild principle for $\left(S_{5}, \phi_{5}, \phi_{6}\right)$.}

The Cayley-Weber type. For our introductory example, we take the type $\left(S_{5}, \phi_{5}, \phi_{6}\right)$, where $\phi_{5}$ is the character of the given degree five permutation representation, and $\phi_{6}$ is the character of the degree six representation $S_{5} \stackrel{\sim}{\longrightarrow} \mathrm{PGL}_{2}(5) \subset S_{6}$. A pair of algebras $\left(K_{5}, K_{6}\right)$ has type $\left(S_{5}, \phi_{5}, \phi_{6}\right)$ exactly when $K_{6}$ is the Cayley-Weber resolvent of $K_{5}$ (see [Jensen et al. 2002, §2.3], for instance, for this notion). An explicit example over $\mathbb{Q}$ is given by $K_{n}=\mathbb{Q}[x] / f_{n}(x)$ with

$$
\begin{array}{ll}
f_{5}(x)=x^{5}-2 x^{4}+4 x^{3}-4 x^{2}+2 x-4, & D_{5}=2^{8} 3^{4} 5^{1}, \\
f_{6}(x)=x^{6}-2 x^{5}+4 x^{4}-4 x^{3}+2 x^{2}-4 x-6, & D_{6}=2^{10} 3^{4} 5^{3} .
\end{array}
$$




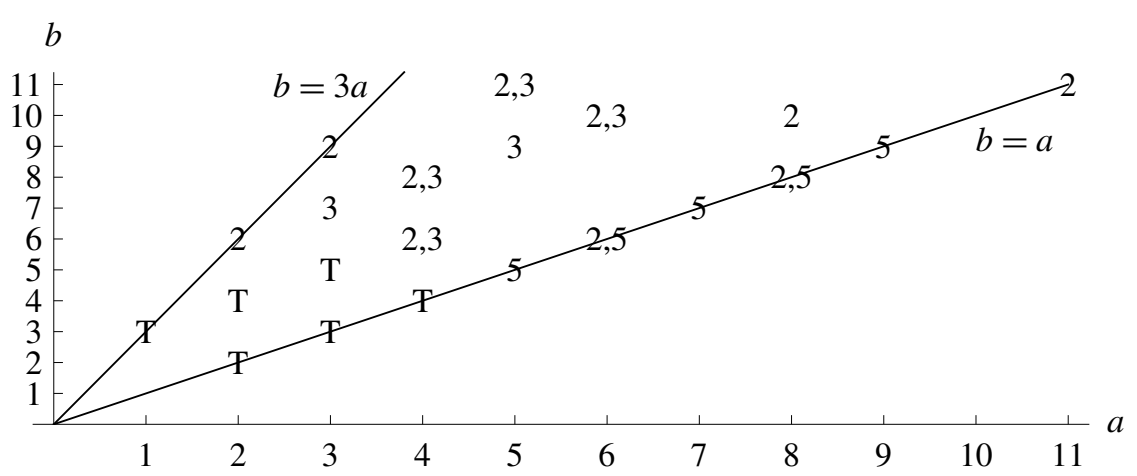

Figure 1. The complete list of pairs $(a, b) \neq(0,0)$ which occur as $\left(a_{\mathfrak{p}}, b_{\mathfrak{p}}\right)$ for $\left(S_{5}, \phi_{5}, \phi_{6}\right)$ over $\mathbb{Q}$. The pairs labeled $\mathrm{T}$ can occur with tame ramification, while the others can only occur for wild $p$-adic ramification as indicated.

In this example, the Galois group is all of $S_{5}$, discriminants $\mathfrak{D}_{K_{n} / \mathbb{Q}}=\left(D_{n}\right)$ are as indicated, and ramification is wild at 2 and tame at 3 and 5 . We are concerned with exponent pairs $\left(a_{\mathfrak{p}}, b_{\mathfrak{p}}\right)$ on discriminants. Here $\left(a_{2}, b_{2}\right)=(8,10),\left(a_{3}, b_{3}\right)=(4,4)$, $\left(a_{5}, b_{5}\right)=(1,3)$, and otherwise $\left(a_{\mathfrak{p}}, b_{\mathfrak{p}}\right)=(0,0)$.

All possibilities for $\left(a_{\mathfrak{p}}, b_{\mathfrak{p}}\right)$. Figure 1 gives all nonzero possibilities for $\left(a_{\mathfrak{p}}, b_{\mathfrak{p}}\right)$ over $\mathbb{Q}$. The fact that the tame list is complete is immediate from the general formalism of the next section. A brute force proof that the wild list is complete goes as follows: there are 113,57 , and 51 quintic algebras $K_{5}$ over $\mathbb{Q}_{p}$ for $p=2$, 3 , and 5 respectively [Jones and Roberts 2006]; for each, one can compute $K_{6}$ and thus the pairs $\left(a_{\mathfrak{p}}, b_{\mathfrak{p}}\right)$; the lists arising are the ones drawn in Figure 1. For larger number fields $F$, the list of possibilities for tame $\left(a_{\mathfrak{p}}, b_{\mathfrak{p}}\right)$ is exactly the same, but the list of possibilities for wild $\left(a_{\mathfrak{p}}, b_{\mathfrak{p}}\right)$ grows without bound.

The tame-wild principle. Figure 1 and the comment about general base fields $F$ clearly illustrate two general phenomena about exponent vectors $\left(a_{\mathfrak{p}}, b_{\mathfrak{p}}\right)$. First, in absolute terms, the exponents can be much larger in wild cases than they are in all tame cases. But second, in relative terms, one can hope that the ratios $a_{\mathfrak{p}} / b_{\mathfrak{p}}$ are quite similar in the wild and tame cases. We are interested in this paper only in the second phenomenon and so we systematically consider ratios.

In our example, the tame-wild principle is the statement that

$$
\frac{1}{3} b_{\mathfrak{p}} \leq a_{\mathfrak{p}} \leq b_{\mathfrak{p}}
$$

holds for all $\left(K_{5}, K_{6}\right) / F$ of type $\left(S_{5}, \phi_{5}, \phi_{6}\right)$ and all primes $\mathfrak{p}$ of $F$. In other words, when $b_{\mathfrak{p}} \neq 0$ one must have $a_{\mathfrak{p}} / b_{\mathfrak{p}} \in\left[\frac{1}{3}, 1\right]$. We have summarized a proof that (2-3) holds when one restricts $F$ to be $\mathbb{Q}$ or one of the $\mathbb{Q}_{p}$. We will see by a 
group-theoretic argument in Section $4 \mathrm{C}$, not involving inspecting wild ramification at all, that (2-3) holds for general $F$. However the situation is subtle, as the analog of (2-3) holds for many $\left(G, \phi_{n}, \phi_{m}\right)$ but not for all.

2B. Application to number field tabulation. The example of this section provides a convenient illustration of the application of tame-wild inequalities to number field tabulation. The right inequality of (2-3) globalizes to the divisibility relation $\mathfrak{D}_{K_{5} / F} \mid \mathfrak{D}_{K_{6} / F}$ which on the level of magnitudes becomes

$$
\left|\mathfrak{D}_{K_{5} / F}\right| \leq\left|\mathfrak{D}_{K_{6} / F}\right| \text {. }
$$

Consider the problem of finding sextic field extensions $K_{6} / F$ with Galois group either $\mathrm{PSL}_{2}(5)$ or $\mathrm{PGL}_{2}(5)$. These all arise as Cayley-Weber resolvents of $K_{5} / F$ with Galois group either $A_{5}$ or $S_{5}$. From (2-4), one sees that to find all $K_{6} / F$ with $\left|\mathfrak{D}_{K_{6} / F}\right| \leq B$ it suffices to find all $K_{5} / F$ with $\left|\mathfrak{D}_{K_{5} / F}\right| \leq B$, apply the Cayley-Weber resolvent, and keep those $K_{6} / F$ with $\left|\mathfrak{D}_{K_{6} / F}\right| \leq B$. This indirect quintic method is enormously faster for large $B$, but the direct sextic method over $F=\mathbb{Q}$ was used in [Ford and Pohst 1992] and [Ford et al. 1998] for the $\mathrm{PSL}_{2}$ (5) and $\mathrm{PGL}_{2}$ (5) cases, respectively.

\section{Character theory and discriminants}

In this section, we review how Artin characters underlie discriminants. Each of the subsections introduces concepts and notation which play an important role in the rest of the paper. The notions we emphasize are slightly different from the most standard representation-theoretic notions. However they are appropriate here because all our characters are rational-valued.

3A. Class sets. Let $G$ be a finite group. We say that two elements of $G$ are power-conjugate if each is conjugate to a power of the other. Let $G^{\sharp}$ be the set of power-conjugacy classes. Thus one has a natural surjection $G \rightarrow G^{\sharp}$, with the fiber $C_{\sigma} \subset G$ above $\sigma \in G^{\sharp}$ being its set of representatives. The order $\bar{\sigma}$ of an element $\sigma \in G^{\sharp}$ is the order of a representing element $g \in C_{\sigma}$. Similarly the power $\sigma^{k}$ of a class $\sigma$ is the class of $g^{k}$ for any representing element $g \in C_{\sigma}$.

When dealing with explicit examples, we most commonly indicate an element of $G^{\sharp}$ by giving its order and an extra identifying label, as in, e.g., 2B. To emphasize the role of order, we say that a class $\tau$ divides a class $\sigma$ if some power of $\sigma$ is $\tau$. Thus divisibility of classes $\tau \mid \sigma$ implies divisibility of integers $\bar{\tau} \mid \bar{\sigma}$, but not conversely. In connection with divisibility, the quantity $[\sigma]=\left|C_{\sigma}\right| / \phi(\bar{\sigma})$ is useful, with $\phi(n)=\left|(\mathbb{Z} / n)^{\times}\right|$the Euler $\phi$-function. This quantity is integral because $C_{\sigma}$ consists of $[\sigma]$ power-classes, each of size $\phi(\bar{\sigma})$. Alternatively, one can think of $G^{\sharp}$ as indexing conjugacy classes of cyclic subgroups of $G$, and then $[\sigma]$ is the number of cyclic subgroups of type $\sigma$. 
Sections 5 and 6 systematically reason with class sets using diagrams based on the divisibility relation and the quantities $[\sigma]$. In general, $G$ itself often recedes into the background of our considerations and the focus is on $G^{\sharp}$ and its inherited structures.

3B. Characters. Our calculations take place mainly in the ring $\mathbb{Q}\left(G^{\sharp}\right)$ of $\mathbb{Q}$-valued functions on $G^{\sharp}$. We also use the larger ring $\mathbb{R}\left(G^{\sharp}\right)$ of real-valued functions, so that we can use standard terms like cone, hull, and interval with their usual meaning. We make extensive use of the natural inner product on $\mathbb{Q}\left(G^{\sharp}\right)$, given by

$$
\left(f_{1}, f_{2}\right)=\sum_{\sigma \in G^{\sharp}} \frac{\left|C_{\sigma}\right|}{|G|} f_{1}(\sigma) f_{2}(\sigma) .
$$

Important elements in $\mathbb{Q}\left(G^{\sharp}\right)$ for us include the characters $\phi_{X}$ of $G$-sets $X$. By definition, these characters are obtained by counting fixed points: $\phi_{X}(\sigma)=\left|X^{g}\right|$, for $g$ any representative of $\sigma$. Both the identity class $e \in G^{\sharp}$ and the constant function $1 \in \mathbb{Q}\left(G^{\sharp}\right)$ usually play trivial roles in our situation. To efficiently remove these quantities from our attention, we define $G^{\sharp 0}=G^{\sharp}-\{e\}$ and let $\mathbb{Q}\left(G^{\sharp}\right)^{0} \subset \mathbb{Q}\left(G^{\sharp}\right)$ be the orthogonal complement to 1 .

The characters $\phi_{G / H}$ and $a_{H}$. Let $H$ be a subgroup of $G$. Then the character of the $G$-set $G / H$ is given by

$$
\phi_{G / H}(\sigma)=\frac{|G|\left|C_{\sigma} \cap H\right|}{|H|\left|C_{\sigma}\right|} .
$$

Taking $H=\{e\}$ gives the regular character $\phi_{G}$ with value $|G|$ at $e$ and 0 elsewhere. We define the formal Artin character of $H$ to then be the difference

$$
a_{H}=\phi_{G}-\phi_{G / H}
$$

which lies in $\mathbb{Q}\left(G^{\sharp}\right)^{0}$. Here we use the adjective "formal" because often one talks about Artin characters only in the presence of fields, while currently we are in a purely group-theoretic setting.

The case that $H$ is cyclic. The case where $H$ is cyclic is particularly important to us. The generators of $H$ all represent the same class $\tau \in G^{\sharp}$ and we use the alternative notation $a_{\tau}=a_{H}$, calling the $a_{\tau}$ tame characters for reasons which will be clear shortly in Section 3C.

To study the $a_{\tau}$ explicitly, it is convenient to make use of what we call precharacters $\hat{a}_{\tau}$, for $\tau \in G^{\sharp}$. By definition, $\hat{a}_{e}$ is the 0 function and otherwise $\hat{a}_{\tau}$ has two nonzero values:

$$
\hat{a}_{\tau}(e)=|G|, \quad \hat{a}_{\tau}(\tau)=-\frac{|G|}{\left|C_{\tau}\right|} .
$$


Tame characters and precharacters are related to each other via

$$
a_{\tau}=\sum_{k \mid \bar{\tau}} \frac{\phi(\bar{\tau} / k)}{\bar{\tau}} \hat{a}_{\tau^{k}}, \quad \hat{a}_{\tau}=\sum_{k \mid \bar{\tau}} \frac{\bar{\tau} \mu(k)}{\phi(\bar{\tau}) k} a_{\tau^{k}},
$$

where $\mu$ is the Möbius $\mu$-function taking values in $\{-1,0,1\}$. Thus, $a_{e}=\hat{a}_{e}=0$, $a_{\tau}=\frac{\bar{\tau}-1}{\bar{\tau}} \hat{a}_{\tau}$ if $\bar{\tau}$ is prime, and otherwise $a_{\tau}$ and $\hat{a}_{\tau}$ are not proportional to each other. As $\tau$ ranges over $G^{\sharp 0}$, the $\hat{a}_{\tau}$ clearly form a basis for $\mathbb{Q}\left(G^{\sharp}\right)^{0}$. So the $a_{\tau}$ also form a basis for $\mathbb{Q}\left(G^{\sharp}\right)^{0}$

3C. Artin characters. Let $F$ be a local or global number field. Let $L / F$ be a Galois extension with Galois group identified with a subgroup of $G$. A permutation representation $\rho$ of $G$ gives an $F$-algebra $K$ split by $L$. For $\mathfrak{p}$ a prime ideal of $F$, the discriminant exponent $c_{\mathfrak{p}}(K)$ depends only on the character $\phi \in \mathbb{Q}\left(G^{\sharp}\right)$ of $\rho$ and in fact depends linearly on $\phi$. The associated Artin character $a_{L / F, \mathfrak{p}}$ is the unique element of $\mathbb{Q}\left(G^{\sharp}\right)$ such that one has the general formula

$$
c_{\mathfrak{p}}(K)=\left(a_{L / F, \mathfrak{p}}, \phi\right) .
$$

From $c_{\mathfrak{p}}(F)=0$, one gets $\left(a_{L / F, \mathfrak{p}}, 1\right)=0$ and so $a_{L / F, \mathfrak{p}} \in \mathbb{Q}\left(G^{\sharp}\right)^{0}$. One can completely compute $a_{L / F, \mathfrak{p}}$ by computing $c_{\mathfrak{p}}(K)$ for any $\left|G^{\sharp 0}\right|$ different $K$ having characters which are linearly independent in $\mathbb{Q}\left(G^{\sharp}\right) / \mathbb{Q}$.

Before continuing, we note a subtlety that disappears in the Artin character formalism that we are reviewing. Namely, it can happen that nonisomorphic algebras $K^{\prime}$ and $K^{\prime \prime}$ give rise to the same permutation character $\phi$. In this case $K^{\prime}$ and $K^{\prime \prime}$ are called arithmetically equivalent. They are indeed equivalent from the point of view of this paper, and any occurrence of $K^{\prime}$ can simply be replaced by $K^{\prime \prime}$.

An Artin conductor $a_{L / F, \mathfrak{p}}$ can be expressed directly in terms of inertia groups in their upper numbering as follows. Let $\mathfrak{P}$ be a prime of $L$ above $\mathfrak{p}$ and let $I_{L / F, \mathfrak{P}} \subseteq \operatorname{Gal}(L / F) \subseteq G$ be the corresponding inertia group. Then one has rational numbers $1 \leq s_{1}<s_{2}<\cdots<s_{k}$ and normal subgroups

$$
I_{L / F, \mathfrak{P}}=I^{s_{1}} \supset I^{s_{2}} \supset \cdots \supset I^{s_{k}} \supset\{e\}
$$

satisfying

$$
a_{L / F, \mathfrak{p}}=\sum_{i=1}^{k}\left(s_{i}-s_{i-1}\right) a_{I^{s_{i}}} .
$$

Here, for the sake of the conciseness of formulas, we put $s_{0}=0$. As a similar convention, we put $I^{s_{k+1}}=\{e\}$. The upper numbers $s_{i}$ we are using here are called slopes in [Jones and Roberts 2006] and are designed to capture tame and wild ramification simultaneously; one has $s_{i}=u_{i}+1$ where the $u_{i}$ are the upper numbers used in the standard reference [Serre 1979]. 
If $s_{1}=1$ then $I^{s_{1}} / I^{s_{2}}$ is cyclic of order prime to $p$. Otherwise, all the $I^{s_{u}} / I^{s_{u+1}}$ are abelian groups of exponent $p$. In particular, $I_{L / F, \mathfrak{P}}$ itself is a $p$-inertial group in the sense that it is an extension of a prime-to- $p$ cyclic group by a $p$-group. In general, we say that a group is inertial if it is $p$-inertial for some prime $p$.

The prime $\mathfrak{p}$ is unramified in $L / F$ if and only if $k=0$ in which case $a_{L / F, \mathfrak{p}}$ is zero. The cases where $\mathfrak{p}$ is ramified but only tamely are those with $k=1$ and $s_{1}=1$. In both these two settings, (3-7) becomes $a_{L / F, \mathfrak{p}}=a_{\tau}$ with $\tau$ being the class of any generator of any $I_{L / F, \mathfrak{P}}$. Thus the tame characters of the previous subsection are exactly the Artin characters which arise when ramification is tame.

3D. Bounds on Artin characters. Define cones in $\mathbb{R}\left(G^{\sharp}\right)^{0}$ spanned by characters or precharacters as follows:

$$
\begin{aligned}
\text { the tame cone } T_{+}(G) & =\left\langle a_{\tau}\right\rangle, \\
\text { the wild cone } W_{+}(G) & =\left\langle a_{L / F, \mathfrak{p}}\right\rangle, \\
\text { the inertial cone } \tilde{T}_{+}(G) & =\left\langle a_{I}\right\rangle, \\
\text { the broad cone } \hat{T}_{+}(G) & =\left\langle\hat{a}_{\tau}\right\rangle .
\end{aligned}
$$

The tame and broad cones are the simplest of these objects, as their generators are indexed by the small and explicit set $G^{\sharp 0}$. The inertial cone is also a purely grouptheoretic object, although now more complicated as its generators are indexed by conjugacy classes of inertial subgroups. Finally, $W_{+}(G)$ is much more complicated in nature: its definition depends on the theory of $p$-adic fields, with $a_{L / F, \mathfrak{p}}$ running over all possible Artin characters, as above.

Our considerations in this section have established the following inclusions:

$$
T_{+}(G) \subseteq W_{+}(G) \subseteq \tilde{T}_{+}(G) \subseteq \hat{T}_{+}(G) .
$$

The first inclusion holds because tame characters are special cases of Artin characters, the second by the expansion (3-7), and the third because all $a_{I}$ take only positive values on $G^{\sharp 0}$.

\section{The tame-wild principle}

We begin in Section 4A by giving a formulation of the tame-wild principle in a somewhat abstract context, so that its motivation and structural features can be seen clearly. Next, Section 4B observes that the bounds from the previous section give techniques for group-theoretically proving instances of the tame-wild principle. Finally, Section 4C details one way of introducing coordinates to render everything explicit and Section 4D sketches alternative approaches.

4A. Abstract formulation. We seek settings where general ramification is governed by tame ramification. The statement that equality holds in $T_{+}(G) \subseteq W_{+}(G)$ 
is true for some $G$, in which case it is the ideal statement. For general $G$, we seek weaker statements in the same spirit. Accordingly, consider the orthogonal projection $a \mapsto a^{V}$ from $\mathbb{R}\left(G^{\sharp}\right)$ onto an arbitrary subspace $V \subseteq \mathbb{R}\left(G^{\sharp}\right)$. Let $T_{+}(G, V), W_{+}(G, V), \tilde{T}_{+}(G, V)$, and $\hat{T}(G, V)$ be the images of $T_{+}(G), W_{+}(G)$, $\tilde{T}_{+}(G)$, and $\hat{T}(G)$, respectively.

Definition 4.1. Let $G$ be a finite group and let $V \subseteq \mathbb{R}\left(G^{\sharp}\right)$ be a subspace. If equality holds in $T_{+}(G, V) \subseteq W_{+}(G, V)$, then we say the tame-wild principle holds for $(G, V)$.

As $V$ gets larger, the tame-wild principle for $(G, V)$ becomes a stronger statement. If it holds when $V$ is all of $\mathbb{R}\left(G^{\sharp}\right)$, then we say it holds universally for $G$.

An important aspect of our formalism is as follows. Given $(G, V)$, consider inertial subgroups $I$ of $G$. For each $I$, one has the subspace $V_{I} \subseteq \mathbb{R}\left(I^{\sharp}\right)$ consisting of pullbacks of functions in $V$ under the natural map $I^{\sharp} \rightarrow G^{\sharp}$. Then the tame-wild principle holds for $(G, V)$ if and only if it holds for all $\left(I, V_{I}\right)$. In fact, while $G$ typically arises as a global Galois group in our applications, whether or not the tamewild principle holds for $(G, V)$ is purely a question about local Galois extensions.

4B. Two proof methods. Projection turns the chain (3-8) into a chain of cones in $V$ :

$$
T_{+}(G, V) \subseteq W_{+}(G, V) \subseteq \tilde{T}_{+}(G, V) \subseteq \hat{T}_{+}(G, V) .
$$

As we will see, for many $G$ all three inclusions are strict in the universal case $V=\mathbb{R}\left(G^{\sharp}\right)$. However strict inclusions can easily become equalities after projection, giving us elementary but quite effective proof techniques. Namely, the broad method for proving that the tame-wild principle holds for $(G, V)$ is to show that equality holds in $T_{+}(G, V) \subseteq \hat{T}_{+}(G, V)$. The inertial method is to show that equality holds in $T_{+}(G, V) \subseteq \tilde{T}_{+}(G, V)$.

Applying the broad method gives the following simple result which we highlight because of its wide applicability:

Theorem 4.2. Let $G$ be a finite group and let $V$ be a subspace of $\mathbb{R}\left(G^{\sharp}\right)$. Suppose that the broad cone $\hat{T}_{+}(G, V)$ is generated by the $\hat{a}_{\tau}^{V}$ with $\tau$ of prime order. Then $T_{+}(G, V)=\hat{T}_{+}(G, V)$ and the tame-wild principle holds for $(G, V)$.

Proof. For $\tau$ of prime order one has

$$
\hat{a}_{\tau}=\frac{\bar{\tau}}{\bar{\tau}-1} a_{\tau},
$$

as noted after (3-4). Thus $\hat{T}_{+}(G, V)$ is contained in $T(G, V)$ and so all four sets in (4-1) are the same.

In general, the broad method is very easy to apply, while the harder inertial method can work when the broad method does not. 
4C. Calculations with permutation characters. Let $\phi_{1}, \ldots, \phi_{r}$ be permutation characters spanning $V$. Then we are exactly in the situation described in the introduction, and in this subsection we describe how one approaches the tame-wild principle in this particular coordinatization. We incorporate the $\phi_{i}$ into our notation in straightforward ways, for example by writing $\left(G, \phi_{1}, \ldots, \phi_{r}\right)$ rather than $(G, V)$.

Throughout this subsection, we illustrate the generalities by returning to the introductory example with $G=S_{5}$ and $V=\left\langle\phi_{5}, \phi_{6}\right\rangle$. The very simple two-dimensional picture of $V$ in Figure 1 serves as an adequate model for mental images of the general situation. In particular, we always think of the $a_{\tau}^{V}, a_{L / F, \mathfrak{p}}, a_{I}^{V}$, and $\hat{a}_{\tau}$ as in the drawn $V$. We think of our various cones in the drawn $V$ as well. On the other hand, it is not useful to draw the $\phi_{i}$ on these pictures. Rather, via the identification of $V$ with its dual by the inner product, we think of the $\phi_{i}$ as coordinate functions on the drawn $V$.

Conductor vectors. The space $V$ is identified with a subspace of $\mathbb{R}^{r}$, viewed but not always written as column vectors, via $v \mapsto\left(c_{1}, \ldots, c_{r}\right)$ with $c_{i}=\left(v, \phi_{i}\right)$. For example, an Artin character $a_{L / F, \mathfrak{p}}^{V}$ becomes a vector of conductors as in the introduction:

$$
c_{L / F, \mathfrak{p}}=\left(c_{\mathfrak{p}}\left(K_{1}\right), \ldots, c_{\mathfrak{p}}\left(K_{r}\right)\right) .
$$

The main case is when the $\phi_{i}$ are linearly independent, so that $V$ is all of $\mathbb{R}^{r}$. One can always work in this main case by picking a basis from among the $\phi_{i}$.

Various matrices. Our approach to calculations centers on matrices. The $r \times G^{\sharp 0}$ partition matrix $P\left(G, \phi_{1}, \ldots, \phi_{r}\right)$ has $i-\tau$ entry the cycle type $\lambda_{\tau}\left(\phi_{i}\right)$ of $\rho_{i}(g)$, where $\rho_{i}$ is a permutation representation with character $\phi_{i}$ and $g \in G$ represents $\tau$. Thus,

$$
P\left(S_{5}, \phi_{5}, \phi_{6}\right)=\left(\begin{array}{cccccc}
2111 & 221 & 311 & 41 & 5 & 32 \\
222 & 2211 & 33 & 411 & 51 & 6
\end{array}\right)
$$

Partition matrices are purely group-theoretic objects, but one can use fields in a standard way to help construct them. For example, the columns from left to right are the partitions obtained by factoring the pair $\left(f_{5}(x), f_{6}(x)\right)$ from (2-1) and (2-2) modulo the primes $67,211,31,13,11$, and 7 respectively.

One passes to the tame matrix $T\left(G, \phi_{1}, \ldots, \phi_{r}\right)$ by replacing each partition $\lambda_{\tau}\left(\phi_{i}\right)$ by its conductor $c_{\tau}\left(\phi_{i}\right)=\left(a_{\tau}, \phi_{i}\right)$ - its degree minus its number of parts. Thus

$$
T\left(S_{5}, \phi_{5}, \phi_{6}\right)=\left(\begin{array}{llllll}
1 & 2 & 2 & 3 & 4 & 3 \\
3 & 2 & 4 & 3 & 4 & 5
\end{array}\right)
$$

The broad matrix $\hat{T}\left(G, \phi_{1}, \ldots, \phi_{r}\right)$ consists of what we call preconductors, the preconductor $\hat{c}_{\tau}\left(\phi_{i}\right)=\left(\hat{a}_{\tau}, \phi_{i}\right)$ being the degree of $\lambda_{\tau}\left(\phi_{i}\right)$ minus its number of ones. 
Thus

$$
\hat{T}\left(S_{5}, \phi_{5}, \phi_{6}\right)=\left(\begin{array}{llllll}
2 & 4 & 3 & 4 & 5 & 5 \\
6 & 4 & 6 & 4 & 5 & 6
\end{array}\right) .
$$

Inertial matrices $\tilde{T}\left(G, \phi_{1}, \ldots, \phi_{r}\right)$ typically have more columns, because columns are indexed by conjugacy classes of inertial subgroups $I$. But an entry is just the formal conductor $c_{I}\left(\phi_{i}\right)=\left(a_{I}, \phi_{i}\right)$, this being the degree of $\rho_{i}$ minus the number of orbits of $\rho_{i}(I)$, just as in the cyclic case. The cones $T_{+} \subseteq \tilde{T}_{+} \subseteq \hat{T}_{+} \subset \mathbb{R}^{r}$ are then generated by the columns of the corresponding matrices $T, \tilde{T}$, and $\hat{T}$.

Inclusions $a^{V} \in T_{+}(G, V)$ in matrix terms. By dropping rows, we can assume that $\phi_{1}, \ldots, \phi_{r}$ span $V$ and so $T=T\left(G, \phi_{1}, \ldots, \phi_{r}\right)$ has full rank $r$, as discussed above. In general, let $c \in \mathbb{R}^{r}$ be a column $r$-vector. For each $r$-element subset $J \subseteq G^{\sharp 0}$ for which the corresponding minor $T(J)$ is invertible, let $u(J)=\left(u(J)_{\tau}\right)_{\tau \in J}$ be the vector $T(J)^{-1} c$. Then $c=\sum_{\tau \in J} u(J)_{\tau} T_{\tau}$, with $T_{\tau}$ the $\tau$-th column of $T$. Then $c$ is in the tame cone $T_{+}$if and only if there exists such a $J$ with $u(J)_{\tau} \geq 0$ for all $\tau \in J$.

To prove that the tame-wild principle holds for $\left(G, \phi_{1}, \ldots, \phi_{r}\right)$ directly, one would have to show this positivity condition holds for all conductor vectors $c_{L / F, \mathfrak{p}}$. To show it via the inertial method, one has to show that it holds for all formal conductor vectors $c_{I}$. To show it holds via the broad method, one has to show that it holds for all preconductor vectors $\hat{c}_{\tau}$.

Projectivization. In the introductory example, we emphasized taking ratios of conductors, thereby removing the phenomenon that wild conductors are typically much larger than tame conductors, but keeping the phenomenon we are interested in. We can do this in the general case as well, assuming without loss of generality that $\phi_{r}$ comes from a faithful permutation representation so that the conductors $c_{\tau}\left(\phi_{r}\right)$ are strictly positive for all $\tau \in G^{\sharp 0}$. We projectivize $c=\left(c_{1}, \ldots, c_{r}\right)$ to $c^{\prime}=\left(c_{1}^{\prime}, \ldots, c_{r-1}^{\prime}\right)$ with $c_{i}^{\prime}=c_{i} / c_{r}$.

Applying this projectivization process to columns gives the projective tame, inertial, and broad matrices respectively, each notationally indicated by $\mathrm{a}^{\prime}$. In our continuing introductory example, one has, very simply,

$$
\begin{aligned}
& T^{\prime}\left(S_{5}, \phi_{5}, \phi_{6}\right)=\left(\begin{array}{llllll}
\frac{1}{3} & 1 & \frac{1}{2} & 1 & 1 & \frac{3}{5}
\end{array}\right), \\
& \hat{T}^{\prime}\left(S_{5}, \phi_{5}, \phi_{6}\right)=\left(\begin{array}{llllll}
\frac{1}{3} & 1 & \frac{1}{2} & 1 & 1 & \frac{5}{6}
\end{array}\right) .
\end{aligned}
$$

In general, the $\tau$-columns of $T^{\prime}\left(G, \phi_{1}, \ldots, \phi_{r}\right)$ and $\hat{T}^{\prime}\left(G, \phi_{1}, \ldots, \phi_{r}\right)$ agree if $\tau$ has prime order. Here they disagree only in the last column corresponding to the composite order 6 .

Let $T_{+}^{\prime}\left(G, \phi_{1}, \ldots, \phi_{r}\right)$ be the convex hull of the columns of $T^{\prime}\left(G, \phi_{1}, \ldots, \phi_{r}\right)$ and define $W_{+}^{\prime}\left(G, \phi_{1}, \ldots, \phi_{r}\right), \tilde{T}_{+}^{\prime}\left(G, \phi_{1}, \ldots, \phi_{r}\right)$ and $\hat{T}_{+}^{\prime}\left(G, \phi_{1}, \ldots, \phi_{r}\right)$ to be the 
analogous hulls. Then (4-1) has its obvious analog at the level of hulls, and one can think about the broad method and the inertial method at this level. In the introductory example, (4-5) and (4-6) say that $T_{+}^{\prime}\left(S_{5}, \phi_{5}, \phi_{6}\right) \subseteq \hat{T}_{+}^{\prime}\left(S_{5}, \phi_{5}, \phi_{6}\right)$ is an equality because both sides are $\left[\frac{1}{3}, 1\right]$. Thus the tame-wild principle holds for $\left(S_{5}, \phi_{5}, \phi_{6}\right)$.

The drop in dimension from $r$ to $r-1$ has a number of advantages. As illustrated already by (4-5) and (4-6), it renders the $r=2$ case extremely concrete. As we will illustrate in Section 8A, it renders the $r=3$ case highly visible. In general, it lets one determine whether $a^{V}$ is in $T_{+}^{\prime}\left(G, \phi_{1}, \ldots, \phi_{r}\right)$ by computation with $(r-1) \times(r-1)$ minors rather than $r \times r$ minors.

4D. Alternative approaches. Our abstract formulation of the tame-wild principle is designed to be very flexible. For example, say that a vector $v \in \mathbb{R}\left(G^{\sharp}\right)$ is bad if $\left(a_{\tau}, v\right) \geq 0$ for all $\tau \in G^{\sharp 0}$ but $\left(a_{L / F, \mathfrak{p}}, v\right)<0$ for some Artin character $a_{L / F, \mathfrak{p}}$. The bad vectors form a union of cones in $\mathbb{R}\left(G^{\sharp}\right)$ and the tame-wild principle holds for $(G, V)$ if and only if $V$ misses all these cones. In this sense, the one-dimensional $V$ spanned by bad $v$ are essential cases, but these $V$ are never spanned by permutation characters.

Sections 5 and 6 are in the universal setting $V=\mathbb{R}\left(G^{\sharp}\right)$, and we do not use $\phi_{i}$ at all. Sections 7 and 8 return to the permutation character setting described in Section 4C. In general, the systematic study of the tame-wild principle for a fixed $G$ and varying $V$ would be facilitated by the canonical basis of $\mathbb{Q}\left(G^{\sharp}\right)$ given by irreducible rational characters.

\section{The universal tame-wild principle holds for U-groups}

In Section 5A, we present a diagrammatic way of understanding class sets $G^{\sharp}$. Making use of this viewpoint, Section 5B gives the canonical expansion of a formal Artin character $a_{I}$ as a sum of tame characters $a_{\tau}$. Next, Section 5C introduces the notion of U-group and proves that the universal tame-wild principle holds for U-groups. However the class of U-groups is quite small, as discussed in Section 5D.

5A. Divisibility posets. For $G$ a finite group, the set $G^{\sharp}$ is naturally a partially ordered set via the divisibility relation. We draw this divisibility poset in the standard way with an edge from $\sigma$ down to $\tau$ of vertical length one if $\sigma^{p}=\tau$ for some prime $p$. With notation as in Section 3A, the natural weight $d(\tau, \sigma)=[\sigma] /[\tau]$ plays an important role, and we write it next to the edge whenever it is different from 1 , considering this data as part of the divisibility poset.

The product of the edge weights from any vertex $\sigma$ down to another $\tau$ is pathindependent, being in fact just $d(\tau, \sigma)=[\sigma] /[\tau]$. Define integers $u_{G, \sigma}$ via

$$
\sum_{\tau \mid \sigma} d(\tau, \sigma) u_{G, \sigma}=1
$$


Thus $u_{G, \tau}=1$ for maximal $\tau$, and all the integers $u_{G, \tau}$ can be computed by downwards induction on the divisibility poset $G^{\sharp}$.

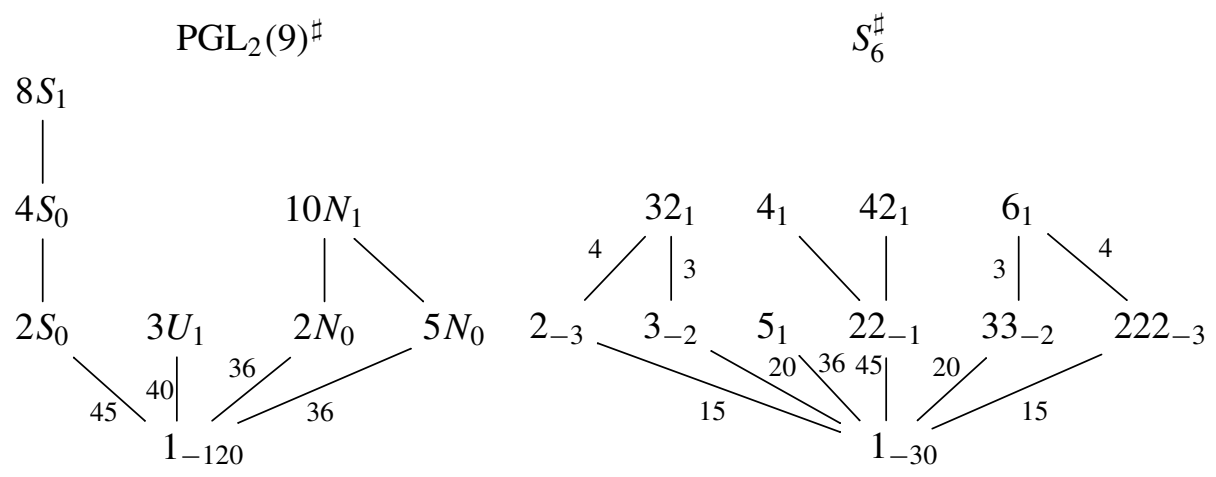

Figure 2. Two divisibility posets $G^{\sharp}$ with $u_{G, \tau}$ subscripted on $\tau \in G^{\sharp}$.

Figure 2 draws the divisibility posets $\mathrm{PGL}_{2}(9)^{\sharp}$ and $S_{6}^{\sharp}$, with each $\tau$ subscripted by its $u_{G, \tau}$. The case $\mathrm{PGL}_{2}(9)$ represents the general case $\mathrm{PGL}_{2}\left(p^{f}\right)$, with split-torus classes indexed by nonunital divisors of $p^{f}-1$, a unipotent class $p U$, nonsplit-torus classes indexed by nonunital divisors of $p^{f}+1$, and finally the identity class 1 . The case $S_{6}$ represents the general case $S_{n}$, where classes are indexed by partitions of $n$, with $1 \mathrm{~s}$ usually left unprinted.

In general, the largest edge weights on divisibility posets $G^{\sharp}$ tend to be on edges incident on the identity class. These edges do not play an important role for us and in the sequel we work instead with the divisibility poset associated to $G^{\sharp 0}$.

5B. Expansion of formal Artin characters. Divisibility posets for inertial groups $I$ and the associated integers $u_{I, \sigma}$ are important to us because of the role they play in the following lemma.

Lemma 5.1. Let $G$ be a finite group, let $I$ be a subgroup, and let $i: I^{\sharp} \rightarrow G^{\sharp}$ be the induced map. Then the expansion of the formal Artin character $a_{I} \in \mathbb{Q}\left(G^{\sharp}\right)^{0}$ in the basis $\left\{a_{\tau}\right\}_{\tau \in G^{\sharp 0}}$ can be read off from the divisibility poset $I^{\sharp 0}$ via the formula

$$
a_{I}=\frac{1}{|I|} \sum_{\sigma \in I^{\sharp 0}} u_{I, \sigma}[\sigma] \bar{\sigma} a_{i(\sigma)} .
$$

Before proving the lemma, we explain the roles that various parts of (5-2) play in the sequel. The positive integer $[\sigma] \bar{\sigma}$ plays a very passive role: only the positivity of $[\sigma] \bar{\sigma}$ is used in the proof of Theorem 5.3; moreover, $[\sigma] \bar{\sigma}$ factors out in Section 6A and accordingly does not enter into Section 6B-Section $6 \mathrm{E}$. The factor $|I|^{-1}$ is more important: while only its positivity enters into the proof of Theorem 5.3, 
it contributes to the index factor in (6-1) which enters significantly into the rest of Section 6. The part with the most important role is $u_{I, \sigma}$, as it is the possible negativity of $u_{I, \sigma}$ that can lead to failures of the tame-wild principle. Our use of the function $i$ relegates the difference between $I$ and $G$ to the background, but one should note that for $\tau \in G^{\sharp 0}$ the actual coefficient of $a_{\tau}$ in (5-2) has $\left|i^{-1}(\tau)\right|$ terms.

Proof. First consider the case $I=G$. Then both sides of (5-2) are in $\mathbb{Q}\left(I^{\sharp}\right)^{0}$. The left side takes the value $a_{I}(\tau)=-1$ for all $\tau \in I^{\sharp 0}$. We thus need to evaluate the right side on an arbitrary $\tau \in I^{\sharp 0}$ and see that it simplifies to -1 :

$$
\begin{aligned}
\frac{1}{|I|} \sum_{\sigma \in I^{\sharp 0}} u_{I, \sigma}[\sigma] \bar{\sigma} a_{\sigma}(\tau) & =\frac{1}{|I|} \sum_{\tau \mid \sigma} u_{I, \sigma}[\sigma] \bar{\sigma} a_{\sigma}(\tau) \\
& =\frac{1}{|I|} \sum_{\tau \mid \sigma} u_{I, \sigma}[\sigma] \bar{\sigma}\left(-\frac{\phi(\bar{\tau})}{\bar{\sigma}} \frac{|I|}{\left|C_{\tau}\right|}\right) \\
& =-\sum_{\tau \mid \sigma} u_{I, \sigma}[\sigma] \frac{\phi(\bar{\tau})}{\left|C_{\tau}\right|} \\
& =-\sum_{\tau \mid \sigma} \frac{u_{I, \sigma}[\sigma]}{[\tau]} \\
& =-\sum_{\tau \mid \sigma} d(\tau, \sigma) u_{I, \sigma}=-1 .
\end{aligned}
$$

Here we have used formulas from Section 3A and Section 3B as well as the definition of $d(\tau, \sigma)$ and the defining property of the $u_{I, \sigma}$ from Section 5A. Finally the case of general $G$ follows, by induction of both sides from $I$ to $G$.

5C. Applying the inertial method. Say that a class $\tau \in G^{\sharp}$ is a $U$-class if it divides exactly one maximal element $\sigma$ of $G^{\sharp}$ and $d(\tau, \sigma)=1$. Otherwise, say it is an $N$-class. Here $\mathrm{U}$ stands for unique and $\mathrm{N}$ for nonunique. The following three facts are immediate from the definition. First, a maximal class $\tau$ is always a U-class with $u_{G, \tau}=1$. Second, other U-classes $\tau$ have $u_{G, \tau}=0$. Third, a maximal N-class $\tau$ always has $u_{G, \tau}<0$.

We divide all finite groups into two types, as follows.

Definition 5.2. A finite group is a $U$-group if every nonidentity element is contained in exactly one maximal cyclic subgroup. Otherwise it is an $\mathrm{N}$-group.

It is immediate that a group $G$ is a $\mathrm{U}$-group if and only if all classes $\tau \in G^{\sharp 0}$ are U-classes. Thus from Figure $2, \mathrm{PGL}_{2}(9)$ is a U-group while $S_{6}$ is an $\mathrm{N}$-group. It follows easily from the definition that any subgroup of a U-group is itself a U-group. Similarly, any quotient of a U-group is a U-group [Suzuki 1950]. 
Via (3-4), the chain (3-8) completely collapses to the equality $T_{+}(G)=\hat{T}_{+}(G)$ if and only if all nonidentity elements in $G$ have prime order. The following theorem is a subtler version of this idea.

Theorem 5.3. Suppose $G$ is a group such that all inertial subgroups of $G$ are $U$-groups. Then one has $T_{+}(G)=\tilde{T}_{+}(G)$, and so the tame-wild principle holds universally for $G$.

Proof. Let $I$ be an arbitrary inertial subgroup. Since $I$ is assumed to be a U-group, the associated integers $u_{I, \sigma}$ are nonnegative for all $\sigma \in I^{\sharp 0}$. For any $\tau \in G^{\sharp 0}$, the terms $|I|^{-1} u_{I, \sigma}[\sigma] \bar{\sigma}$ contributing to the coefficient of $a_{\tau}$ in Lemma 5.1 are all nonnegative. Hence the coefficient itself is nonnegative and so $a_{I}$ is in the tame cone $T_{+}(G)$. Since the $a_{I}$ generate the inertial cone $\tilde{T}_{+}(G)$, equality holds in $T_{+}(G) \subseteq \tilde{T}_{+}(G)$.

In particular, the tame-wild principle holds for all U-groups. This is the main import of Theorem 5.3, as we are not aware of any group satisfying the hypothesis of Theorem 5.3 which is not itself a U-group.

5D. Classification of $\boldsymbol{U}$-groups. Given Theorem 5.3, it is of interest to classify U-groups. This problem has been addressed in the literature, with Kontorovich [1939; 1940] referring to U-groups as completely decomposable groups, and Suzuki [1950] calling them groups with a complete partition. We give a summary of the classification situation here.

The condition to be a U-group is very restrictive, but it is easy to check that it includes many groups of small order. In particular, the following groups are Ugroups: cyclic groups, dihedral groups, groups of prime exponent, and the Frobenius groups $F_{p}=C_{p}: C_{p-1}$. The last class is particularly important in our context, since an extension of a $p$-adic field of degree $p$ has normal closure with Galois group a subgroup of $F_{p}$. If $q$ is a prime power, the linear groups $\operatorname{PSL}_{2}(q)$ and $\mathrm{PGL}_{2}(q)$ are U-groups, so that in particular $S_{4} \cong \mathrm{PGL}_{2}(3), S_{5} \cong \mathrm{PGL}_{2}(5)$ and $A_{6} \cong \mathrm{PSL}_{2}$ (9) are all U-groups. There are more U-groups than those listed here, most of them being more general types of Frobenius groups.

The following observation is useful in understanding the nature of U-groups. In two settings, the extreme members of a class of groups are exactly the U-groups as follows. First, consider abelian $p$-groups of order $p^{n}$. Up to isomorphism, they correspond to partitions of $n$. The groups which are U-groups are the two extreme ones $\left(C_{p}\right)^{n}$ and $C_{p^{n}}$. Second, consider semidirect products $C_{a}:_{\gamma} C_{b}$ with $a$ and $b$ being relatively prime and $\gamma: C_{b} \rightarrow \operatorname{Aut}\left(C_{a}\right)$. If $\gamma$ is trivial, then $C_{a}:{ }_{\gamma} C_{b} \cong C_{a b}$ is a U-group. If $\gamma$ is injective, then $C_{a}:_{\gamma} C_{b}$ is again a U-group, being of a nature similar to $F_{p}$ above. Again, it is the intermediate cases which are N-groups: if $\gamma$ is neither trivial nor injective then nontrivial elements in the kernel of $\gamma$ are in more than one maximal cyclic subgroup. 


\section{The universal tame-wild principle usually fails for N-groups}

In this section, we study the universal tame-wild principle for $\mathrm{N}$-groups. In Section 6A, we give the canonical expansion of a general Artin character $a_{L / F, \mathfrak{p}}$ in terms of tame characters $a_{\tau}$. In Section 6B, we list out N-groups of order $p q r$ where $p, q$, and $r$ are not necessarily distinct primes, finding six series. We show in Section 6C that the universal tame-wild principle generally fails for groups in the first four series. In Section 6D we take a close look at the quaternion group $Q_{8}$, which is the first group of the fifth series, finding failure again. On the other hand we show in Section $6 \mathrm{E}$ that the universal tame-wild principle holds for all groups in the sixth series. Finally, Section 6F explains how the negative results for small groups support the principle that most $\mathrm{N}$-groups do not satisfy the universal tame-wild principle.

6A. Expansion of general Artin characters. Let $a_{L / F, \mathfrak{p}} \in \mathbb{Q}\left(G^{\sharp}\right)^{0}$ be an Artin character coming from an inertial subgroup $I \subseteq G$. Equation (3-7) expands $a_{L / F, \mathfrak{p}}$ in terms of formal Artin characters $a_{I^{s_{i}}}$ and Lemma 5.1 in turn expands each $a_{I^{s_{i}}}$ in terms of tame characters. Putting these two expansions together and replacing the divisibility posets $\left(I^{s_{i}}\right)^{\sharp 0}$ with their images in $I^{\sharp 0}$ gives the following lemma.

Lemma 6.1. Let $G$ be a group, and let $a_{L / F, \mathfrak{p}} \in \mathbb{Q}\left(G^{\sharp}\right)^{0}$ be an Artin character with inertia group $I=I^{s_{1}} \supset I^{s_{2}} \supset \cdots$ as in (3-6). Let $i: I^{\sharp} \rightarrow G^{\sharp}$ be the induced map. Then one has the expansion

$$
a_{L / F, \mathfrak{p}}=\frac{1}{|I|} \sum_{\sigma \in I^{\sharp 0}} w_{L / F, \mathfrak{p}, \sigma}[\sigma] \bar{\sigma} a_{i(\sigma)},
$$

where

$$
w_{L / F, \mathfrak{p}, \sigma}=\sum_{i=1}^{k}\left(s_{i}-s_{i-1}\right)\left[I: I^{s_{i}}\right] u_{I^{s_{i}}, \sigma} .
$$

While the lemma applies to the general situation, our focus in Sections $6 \mathrm{~B}-6 \mathrm{E}$ is on the case $I=G$. Here $a_{L / F, \mathfrak{p}}$ is in the tame cone $T_{+}(G)$ if and only if $w_{L / F, \mathfrak{p}, \tau} \geq 0$ for all $\tau \in G^{\sharp 0}$.

6B. Inertial $\mathbf{N}$-groups of order pqr. Groups of order $p$ or $p q$ are U-groups. In the complete list of inertial groups of order $p q r$, in a rough sense about half of them are $\mathrm{U}$-groups and the other half $\mathrm{N}$-groups. For example, for a given prime $p$, there are two nonabelian groups of order $p^{3}$, the extra-special groups often denoted $p_{+}^{1+2}$ and $p_{-}^{1+2}$. For $p$ odd, $p_{+}^{1+2}$ has exponent $p$ and so is a U-group, while $p_{-}^{1+2}$ is an N-group. Similarly the dihedral group $D_{4}=2_{+}^{1+2}$ is a U-group while the quaternion group $Q_{8}=2_{-}^{1+2}$ is an N-group. 
In fact, it is easy to see that the inertial N-groups are as follows. Now $p, q$, and $r$ are required to be different primes, with $q \mid p-1$ whenever $F_{p, q}=C_{p}: C_{q}$ is present:

1: The product $F_{p, q} \times C_{r}$.

2: The semidirect product $C_{p}: C_{q^{2}} \cong F_{p, q} *_{C_{q}} C_{q^{2}}$.

3: The abelian group $C_{p q} \times C_{p} \cong C_{p} \times C_{p} \times C_{q}$.

4: The product $F_{p, q} \times C_{p}$.

5: The extra-special group $p_{-}^{1+2}$.

6: The abelian group $C_{p^{2}} \times C_{p}$.

These groups $I$ are all $p$-inertial groups, but not inertial groups for any other primes. Moreover, since all proper subgroups are U-groups, the universal tamewild principle fails for $I$ if and only if there exists a totally ramified local Galois extension $L / F$ having $\operatorname{Gal}(L / F) \cong I$ with associated Artin character $a_{L / F, \mathfrak{p}}$ having a negative coefficient $w_{L / F, \mathfrak{p}, \tau}$. Furthermore, in each case it turns out that there is exactly one $\mathrm{N}$-class $\tau \in I^{\sharp 0}$. Only for this class $\tau$ could $w_{L / F, \mathfrak{p}, \tau}$ possibly be negative, and this $\mathrm{N}$-class is boxed in the displayed divisibility posets below.

In general, let $I$ be a $p$-inertial group. Then it is known that there indeed exists a totally ramified Galois extension of $p$-adic fields $L / F$ with $\mathrm{Gal}(L / F)$ isomorphic to $I$. This fact for our particular $I$ is essential to our proofs that the universal tame-wild principle does not hold. However it is easy to prove this fact for all the above $I$ by direct exhibition of $L / F$. We will go into this level of detail only for the groups in Series 4 and $Q_{8}$ from Series 5, as here we need particular fields satisfying conditions on their wild ramification.

6C. Negative results for four series. Our first result concerns Series 1-4 and is negative:

Theorem 6.2. $F_{3,2} \times C_{3} \cong S_{3} \times C_{3}$ satisfies the universal tame-wild principle, but otherwise the groups $F_{p, q} \times C_{r}, C_{p}: C_{q^{2}}, C_{p q} \times C_{p}$, and $F_{p, q} \times C_{p}$, do not.

Proof. In the divisibility posets below, the wild classes, meaning the classes of $p$-power order, are put in boldface for further emphasis. For the first three series, the unique N-class $\tau$ has prime-to- $p$ order and so we do not need to enter into an examination of wild slopes. In Series $4, \tau$ has order $p$ and bounds on wild slopes lead to the exception.

1. For the group $I=F_{p, q} \times C_{r}$, power-conjugacy classes are determined by their orders, and the divisibility poset $I^{\sharp 0}$ is 


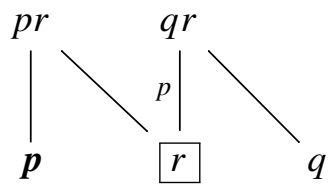

Equation (6-1) becomes $w_{r}=u_{I, r}=-p<0$. So by the existence of totally ramified $I$-extensions as discussed in the previous subsection, the universal tamewild principle does not hold for $I=F_{p, q} \times C_{r}$.

2. The group $I=C_{p}: C_{q^{2}}$ behaves very similarly. Again power-conjugacy classes are determined by their orders:

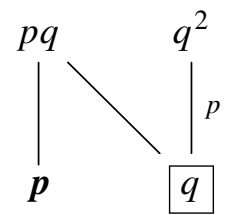

The key quantity $w_{q}=u_{I, q}=-p$ is again negative, so the universal tame-wild principle fails for $C_{p}: C_{q^{2}}$.

3. The group $I=C_{p}^{2} \times C_{q}$ has a more complicated divisibility poset $I^{\sharp 0}$ but the behavior is otherwise similar. The classes of order $p$ and the classes of order $p q$ have the structure of projective lines over $\mathbb{F}_{p}$ in bijection with one another:

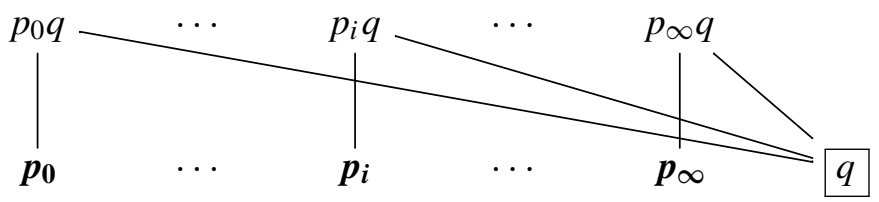

Once again $w_{q}=u_{I, q}=-p$ and so the universal tame-wild principle fails for $C_{p}^{2} \times C_{q}$.

4. For $I=F_{p, q} \times C_{p}$ the divisibility poset $I^{\sharp 0}$ is disconnected:

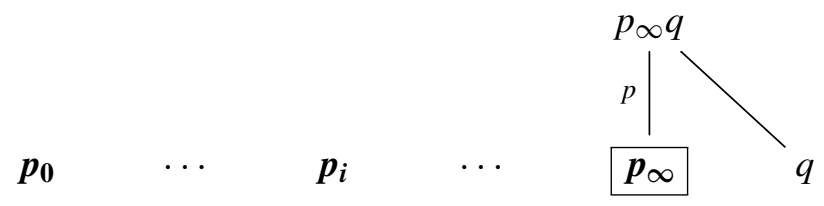

Here $p_{0}$ and $q$ lie in the factor $F_{p, q}$ while $p_{\infty}$ lies in the factor $C_{p}$. The first term in (6-1) is $u_{I, p_{\infty}}=1-p$. However, now we must take into account how wild ramification contributes to the remaining terms. Let $s>1$ be the slope associated to $F_{p, q}$ and let $c>1$ be the slope associated to $C_{p}$. Since $C_{p}$ is abelian, $c$ must be integral and hence $c \geq 2$. On the other hand, $s$ must have exact denominator $q$. Let 
$m=\min (c, s)$, so that $I^{m}=C_{p}^{2}$ is the wild inertia group and $I^{\max (c, s)} \cong C_{p}$ is a higher inertia group. If $c>s$ then $\left(I^{c}\right)^{\sharp 0}=\left\{p_{\infty}\right\}$, while if $s>c$ then $\left(I^{s}\right)^{\sharp 0}=\left\{p_{0}\right\}$. Equation (6-1) becomes

$$
w_{p_{\infty}}= \begin{cases}(1-p)+q(s-1)+q p(c-s) & \text { if } c>s, \\ (1-p)+q(c-1) & \text { if } s>c .\end{cases}
$$

For $(p, q)=(3,2)$, the general formula simplifies to

$$
w_{3_{\infty}}= \begin{cases}6 c-4 s-4 & \text { if } c>s, \\ 2 c-4 & \text { if } s>c .\end{cases}
$$

Thus, using $c \geq 2$, one has $w_{3_{\infty}} \geq 0$ and so the universal tame-wild principle holds for $F_{3,2} \times C_{3}$.

There are many ways to produce an explicit instance with $w_{p_{\infty}}<0$ for the remaining $(p, q)$. We will present one in the setting $s>c=2$ in which case $w_{p_{\infty}}=1+q-p$ is indeed negative. To get an $F_{p, q}$ extension, start with $x^{p}-p$, which gives a totally ramified $F_{p, p-1}$ extension of $\mathbb{Q}_{p}$ with wild slope best written in the form $1+p /(p-1)$. Write $e=(p-1) / q$ and extend the ground field from $\mathbb{Q}_{p}$ to $F_{e}=\mathbb{Q}_{p}[\pi] /\left(\pi^{e}-p\right)$. Then $x^{p}-p$ has Galois group $F_{p, q}$ over $F_{e}$, with wild slope $1+e p /(p-1)$, as tame base-change always scales slopes this way. But now $x^{p}-\pi x^{p-1}+\pi$ has wild slope 2 and, after perhaps replacing $F_{e}$ by an unramified extension $F$, Galois group $C_{p}$ [Amano 1971]. The splitting field of $\left(x^{p}-p\right)\left(x^{p}-\pi x^{p-1}+\pi\right)$ gives the desired extension $L / F$, showing that the universal tame-wild principle does not hold for $F_{p, q} \times C_{p}$.

6D. Negative result for $Q_{8}$. The fifth series, consisting of groups of the form $p_{-}^{1+2}$, is the most complicated. Here we treat only $2_{-}^{1+2}=Q_{8}$, getting a negative result.

Proposition 6.3. The universal tame-wild principle fails for the quaternion group. Proof. The divisibility poset $Q_{8}^{\sharp 0}$, with unique N-class boxed as always, is

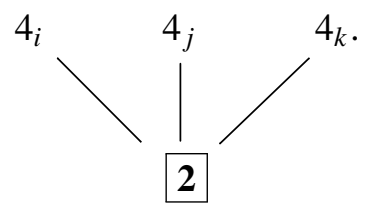

The generic case has three distinct slopes. We seek only counterexamples and so we focus on the special case with two slopes $s_{1}<s_{2}$, with $s_{1}$ occurring with multiplicity two. The key quantity (6-1) here becomes $w_{2}=-2 s_{1}+4\left(s_{2}-s_{1}\right)=4 s_{2}-6 s_{1}$. Thus one gets a counterexample to the universal tame-wild principle if and only if $s_{2}<1.5 s_{1}$.

The table of octic 2-adic fields [Jones and Roberts 2008] available from the website of [Jones and Roberts 2006] then give four types of counterexamples in this 
context, after tame base-change from $\mathbb{Q}_{2}$ to its Galois extension $F$ with ramification index $t$ and residual field degree $u$ :

\begin{tabular}{ccccccc}
$\#$ & $c$ & slope content & $I$ & $D$ & $s_{1}$ & $s_{2}$ \\
\hline 2 & 10 & {$[1 . \overline{3}, 1 . \overline{3}, 1.5]_{3}^{2}$} & $\mathrm{SL}_{2}(3)$ & $\mathrm{GL}_{2}(3)$ & 2 & 2.5 \\
2 & 16 & {$[2,2,2.5]^{2}$} & $Q_{8}$ & $\hat{Q}_{8}$ & 2 & 2.5 \\
4 & 16 & {$[2,2,2.5]^{4}$} & $Q_{8}$ & $8 \mathrm{~T} 17$ & 2 & 2.5 \\
8 & 22 & {$[2 . \overline{6}, 2 . \overline{6}, 3.5]_{3}^{2}$} & $\mathrm{SL}_{2}(3)$ & $\mathrm{GL}_{2}(3)$ & 6 & 8.5
\end{tabular}

Here in the first and last cases, we use the general conversion from slope content $\left[\ldots \sigma_{i} \ldots\right]_{t}^{u}$ over $\mathbb{Q}_{2}$ to slope content $\left[\ldots s_{i} \ldots\right]_{1}^{1}$ over $F$ given by $s_{i}=1+t\left(\sigma_{i}-1\right)$. A full treatment of the range of possible counterexamples could have Proposition 4.4 of [Fontaine 1971] as its starting point.

Our counterexamples in Section 7D and Section 8B will be built from one of the two fields with slope content $[2,2,2.5]^{2}$. A point to note here is that $\mathbb{Q}_{2}$ does have totally ramified quaternionic extensions, in fact four of them, all with slope content [2, 3, 4] [Jones and Roberts 2008]. However, these extensions do not give counterexamples to the universal tame-wild principle for $Q_{8}$. The fact that the first local counterexamples come from $\hat{Q}_{8}=8 \mathrm{~T} 8$ extensions of $\mathbb{Q}_{2}$ plays a prominent role in our later global counterexamples.

6E. Positive results for $\boldsymbol{C}_{\boldsymbol{p}^{2}} \times \boldsymbol{C}_{\boldsymbol{p}}$. Here we prove that the N-groups in Series 6 always satisfy the universal tame-wild principle. Unlike most of our previous positive results, but like the exception $S_{3} \times C_{3}$ of Section $6 \mathrm{C}$, this result is not purely group-theoretic. Rather it depends on a close analysis of the possibilities for wild slopes. Said in a different way, the situation for these $I$ is $T_{+}(I)=W_{+}(I) \subset \tilde{T}_{+}(I)$, so that the universal tame-wild principle holds, even though it is not provable by the inertial method.

Theorem 6.4. The groups $C_{p^{2}} \times C_{p}$ satisfy the universal tame-wild principle.

Proof. Let $K_{p^{2}} / F$ be a cyclic extension of degree $p^{2}$ and slopes $s_{1}<s_{2}$. Let $K_{p} / F$ be a cyclic extension of degree $p$ and slope $t$. Switch to the indexing scheme of [Serre 1979] via $s_{i}=1+v_{i}$ and $t=1+c$, so as to better align also with our reference [Fontaine 1971] and in particular make (6-5) below as simple as possible. There are three possibilities for how the slope filtration goes through the group:

$c<v_{1}<v_{2}$

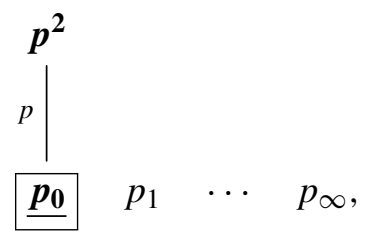

$v_{1} \leq c<v_{2}$

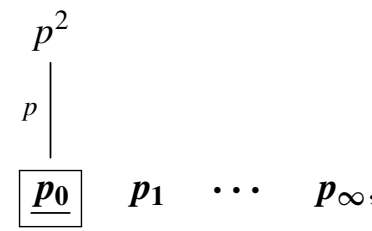

$v_{1}<v_{2} \leq c$ 
Here, assuming all inequalities are strict, classes in the higher inertia group of order $p^{2}$ are put in bold and the classes in the higher inertia group of order $p$ are furthermore underlined. If one has equality, the formulas below still apply.

As in the previous two subsections, only one $w_{\tau}$ from Lemma 6.1 could possibly be negative, and in this case it is $w=w_{p_{0}}$. Equation (6-1) becomes

$$
w= \begin{cases}(c+1)(1-p)+\left(v_{2}-v_{1}\right) p^{2} & \text { if } c<v_{1}<v_{2}, \\ \left(v_{1}+1\right)(1-p)+\left(c-v_{1}\right) p+\left(v_{2}-c\right) p^{2} & \text { if } v_{1} \leq c<v_{2}, \\ \left(v_{1}+1\right)(1-p)+\left(v_{2}-v_{1}\right) p & \text { if } v_{1}<v_{2} \leq c .\end{cases}
$$

Let $e$ be the ramification index of $F / \mathbb{Q}_{2}$ and put $B=e /(p-1)$. From the known behavior of cyclic degree $p$ extensions, one has

$$
1 \leq c \leq p B \quad \text { and } \quad 1 \leq v_{1} \leq p B .
$$

There are two regimes to consider: the geometric regime, where $v_{1}<B$, and the arithmetic regime, where $v_{1} \geq B$. One has

$$
\begin{array}{ll}
v_{2} \geq p v_{1} \quad \text { in the geometric regime, } \\
v_{2}=v_{1}+e \quad \text { in the arithmetic regime. }
\end{array}
$$

These last two facts and other related information dating back to [Maus 1965] are conveniently available in [Fontaine 1971, Proposition 4.3].

The quantity $e$ does not enter into the geometric inequality (6-5), and since we need to deal with arbitrary $e$ the upper bounds in (6-4) are not available to us. This fact is the source of our terminology because the geometric case is now identified with the case where $p$-adic fields have been replaced by $\mathbb{F}_{p^{f}}((t))$, which have $e=\infty$. The worst case is always when $v_{2}=p v_{1}$ and, in the second case, when $c$ takes on its limiting bound $v_{2}$ as well. Substituting these worst cases into (6-3) and simplifying, one has

$$
w \geq \begin{cases}\left(v_{1}+1\right)(1-p)+\left(p v_{1}-v_{1}\right) p^{2} & \text { if } c<v_{1}<v_{2}, \\ \left(v_{1}+1\right)(1-p)+\left(p v_{1}-v_{1}\right) p & \text { if } v_{1} \leq c<v_{2}, \\ \left(v_{1}+1\right)(1-p)+\left(p v_{1}-v_{1}\right) p & \text { if } v_{1}<v_{2} \leq c .\end{cases}
$$

With $m$ equal to $p^{2}, p, p$ in the three cases, one further simplifies by

$$
w \geq(p-1)\left(-v_{1}-1+v_{1} m\right)=(p-1)\left((m-1) v_{1}-1\right) \geq 0 .
$$

Thus, in the geometric regime, $w$ is never negative.

In the arithmetic regime the substitute (6-6) for (6-5) is simpler in that it is an equality, but now the upper bounds in (6-4) will need to be used. The substitution $v_{2}=v_{1}+e=v_{1}+B(p-1)$ into (6-3) makes $w$ factor, and we divide by the positive 
quantity $p-1$ :

$$
\frac{w}{p-1}= \begin{cases}B p^{2}-1-c & \text { if } c<v_{1}<v_{1}+e \\ B p^{2}-v_{1}-p\left(c-v_{1}\right)-1 & \text { if } v_{1} \leq c<v_{1}+e \\ B p-v_{1}-1 & \text { if } v_{1}<v_{1}+e \leq c\end{cases}
$$

Using the bounds (6-4) one has

$$
\frac{w}{p-1} \geq\left\{\begin{array}{l}
B p^{2}-1-B p=B p(p-1)-1=e p-1 \geq 1 \\
B p^{2}-B p-p(e-1)-1=e p-p(e-1)-1=p-1 \geq 1 \\
B p-v_{1}-1 \leq B p-(B p-e)-1=e-1 \geq 0
\end{array}\right.
$$

in the three cases. Thus here too $w \geq 0$.

6F. From smaller to larger groups. Our final topic in this section is to promote our counterexamples from the small groups $I$ to larger groups $G$ that contain them. In general, let $I \subseteq G$ be an inclusion of groups and consider the induced map $i: I^{\sharp} \rightarrow G^{\sharp}$. Then the lack of injectivity of $i$ can obstruct the promotion process. For example, consider Series 4 groups $I=F_{p, q} \times C_{p}$ and their product embedding into $G=S_{p^{2}}$. Then all $p+1$ classes in $I^{\sharp}$ of order $p$ go to the single class in $S_{p^{2}}^{\sharp}$ indexed by the partition $p^{p}=p \cdots p$. To get the coefficient of $a_{p^{p}}$ of the pushedforward formal Artin conductor $a_{I} \in \mathbb{Q}\left(S_{p^{2}}^{\sharp}\right)^{0}$ one has to add the contributions of the fiber, as in Lemma 5.1. There are $p$ contributions of $1 / p q$ and one contribution of $(2-p) / p q$ for a total of $2 / p q$. Equation (6-1) says that wild ramification can only increase this $2 / p q$ to larger positive numbers, and so all pushed-forward Artin characters $a_{L / F, \mathfrak{p}}$ from $I$ are in the tame cone $T_{+}\left(S_{p^{2}}\right)$.

For Series 1-3 and also for $Q_{8}$, this complication does not arise because the unique $\mathrm{N}$-class in $I^{\sharp 0}$ is the only class of its order. Hence the promotion process works:

Corollary 6.5. Let $G$ be a group containing a subgroup I of the form $F_{p, q} \times C_{r}$, $C_{p}: C_{q^{2}}, C_{p q} \times C_{p}$ or $Q_{8}$. Then $G$ does not satisfy the universal tame-wild principle.

Since there are so many possibilities for $I$, the hypothesis holds for many $G$. Moreover the fact that it holds for a given $G$ is often easily verified. For example, when studying $G$ one commonly has a list of maximal subgroups $H$, and one can often easily see that at least one $I$ is in at least one of the $H$. As another example, the presence of $C_{p q} \times C_{p}$ can often be read off from the divisibility poset: suppose one has a class $\tau \in G^{\sharp}$ of order $\bar{\tau}=p q$ not dividing a class of order $p^{2} q$ but such that $p^{2}$ divides the numerator of $|G| /\left|C_{\tau}\right|$. Then any representative $g$ of $\tau$ lies in a group of type $C_{p q} \times C_{p}$. This criterion is satisfied particularly often for $p=2$ and some odd prime $q$. 


\section{Comparing an algebra with its splitting field}

In this section we return to a very concrete setting, considering types $\left(G, \phi_{i}, \phi_{r}\right)$ where $\phi_{i}$ comes from a faithful permutation representation $i: G \subseteq S_{n}$ and $\phi_{r}$ is the regular character. Thus we are considering algebras $K=K_{i}$ of a specified Galois type compared with their splitting fields $K^{\mathrm{gal}}=K_{r}$.

In Section 7A we introduce explicit notation for comparing two algebras and in Section 7B we explain how it is sometimes best to highlight root discriminants $\mathfrak{d}$ rather than discriminants $\mathfrak{D}$. The tame-wild principle in the notation set up then takes the following form:

$$
\mathfrak{d}_{K^{\text {gal }} / F}^{\underline{\alpha}\left(G, \phi_{i}, \phi_{r}\right)}\left|\mathfrak{d}_{K / F}\right| \mathfrak{d}_{K^{\text {gal }} / F}^{\underline{\omega}\left(G, \phi_{i}, \phi_{r}\right)} .
$$

We observe in Section $7 \mathrm{C}$ that the right divisibility often trivially holds. In Section 7D, we give four examples where it holds nontrivially and one where it fails to hold. In Section 7E we show that the left divisibility always holds, and discuss applications to number field tabulation.

7A. Generalities. The case $r=2$ of just two algebras deserves special attention for at least three reasons. First, hulls $T_{+}^{\prime}\left(G, \phi_{1}, \phi_{2}\right) \subset \mathbb{R}^{1}$ are intervals while hulls for larger $r$ can have up to $\left|G^{\sharp 0}\right|$ vertices. Second, the inequality for each face of any $T_{+}^{\prime}\left(G, \phi_{1}, \ldots, \phi_{r}\right)$ also comes from some $T_{+}^{\prime}\left(G, \psi_{1}, \psi_{2}\right)$ with the new characters $\psi_{j}$ being certain sums of the old characters $\phi_{i}$. Third, it is the case which applies most directly to number field tabulation.

To present results coming from $r=2$ as explicitly as possible, we let $\alpha=$ $\alpha\left(G, \phi_{1}, \phi_{2}\right)$ and $\omega=\omega\left(G, \phi_{1}, \phi_{2}\right)$ be the left and right endpoints of the interval $T_{+}^{\prime}\left(G, \phi_{1}, \phi_{2}\right)$. The tame-wild principle says that all local exponents satisfy

$$
\alpha c_{\mathfrak{p}}\left(K_{2}\right) \leq c_{\mathfrak{p}}\left(K_{1}\right) \leq \omega c_{\mathfrak{p}}\left(K_{2}\right)
$$

In this $r=2$ setting, the tame-wild principle breaks cleanly into two parts: the left and right tame-wild principles respectively say that the left and right inequalities in (7-1) always hold. Similarly, one has the perhaps larger inertial interval $[\tilde{\alpha}, \tilde{\omega}]$ and the perhaps even larger broad interval $[\hat{\alpha}, \hat{\omega}]$.

To transfer the additive inequalities (7-1) into the multiplicative language of divisibility, we make use of the following formalism. Note that the torsion-free group $\mathscr{I}$ of fractional ideals of a local or global number field $F$ embeds into its tensor product over $\mathbb{Z}$ with $\mathbb{Q}$, a group we write as $g^{\mathbb{Q}}$ to account for the fact that $\Phi$ is written multiplicatively. In $g^{\mathbb{Q}}$, as our notation indicates, general rational exponents on ideals are allowed. Then (7-1) corresponds to

$$
\mathfrak{D}_{K_{2} / F}^{\alpha}\left|\mathfrak{D}_{K_{1} / F}\right| \mathfrak{D}_{K_{2} / F}^{\omega},
$$


which makes sense for both local and global number fields. In this formalism, the relations of the introductory example take the form $\mathfrak{D}_{K_{6} / F}^{1 / 3}\left|\mathfrak{D}_{K_{5} / F}\right| \mathfrak{D}_{K_{6} / F}$.

7B. Mean-root normalization and the comparison interval. It is sometimes insightful to switch to a slightly different normalization. We call this normalization mean-root normalization, with "mean" capturing how additive quantities are renormalized and "root" capturing how multiplicative quantities are renormalized.

If $K / F$ has degree $n$ and discriminant $\mathfrak{D}_{K / F}$ then its root discriminant is by definition $\mathfrak{d}_{K / F}=\mathfrak{D}_{K / F}^{1 / n}$. To make this shift in our formalism, we simply replace all permutation characters $\phi_{i}$ by the scaled-down quantities $\phi_{i}=\phi_{i} / \phi_{i}(1)$. One has mean tame conductors $\underline{c}_{\tau}\left(\phi_{i}\right)=\left(a_{\tau}, \phi_{i}\right)$ as well as their analogs $\underline{c}_{I}\left(\phi_{i}\right)=\left(a_{I}, \phi_{i}\right)$ and $\underline{\hat{c}}_{\tau}\left(\phi_{i}\right)=\left(\hat{a}_{\tau}, \phi_{i}\right)$. We always indicate this alternative convention by underlining. Thus the mean-root normalized tame hull for two characters indexed by dimension is $\underline{T}_{+}^{\prime}\left(G, \phi_{n}, \phi_{m}\right)=[\underline{\alpha}, \underline{\omega}]$ where $\underline{\alpha}=m \alpha / n$ and $\underline{\omega}=m \omega / n$. The divisibility relation (7-2) becomes $\mathfrak{d}_{K_{m} / F}^{\alpha}\left|\mathfrak{d}_{K_{n} / F}\right| \mathfrak{d}_{K_{m} / F}^{\omega}$.

The comparison interval $[\underline{\alpha}, \underline{\omega}]$ just introduced supports an intuitive understanding of how ramification in $K_{n} / F$ and $K_{m} / F$ relate to each other. Suppose, for example, that $K_{m} / K_{n} / F$ is a tower of fields so that one has the standard divisibility relation

$$
\mathfrak{d}_{K_{n} / F} \mid \mathfrak{d}_{K_{m} / F} .
$$

Then, assuming $K_{m} / F$ is actually ramified, the ratio

$$
\frac{\log \left|\mathfrak{d}_{K_{n} / F}\right|}{\log \left|\mathfrak{d}_{K_{m} / F}\right|} \in[0,1]
$$

can be understood as the fraction of ramification in $K_{m} / F$ which is seen already in $K_{n} / F$. If the corresponding tame-wild principle holds, then this quantity is guaranteed to be in $[\underline{\alpha}, \underline{\omega}]$.

The mean-root normalization introduces a sense of absolute scale, with the number one playing a prominent role, as illustrated by the preceding paragraph and the next three subsections. Assuming $\phi_{n}-\phi_{m}$ is not a constant, one always has strict inequality $\underline{\alpha}<\underline{\omega}$. The failure of resolvent constructions from $\left(G, \phi_{n}\right)$ fields to $\left(G, \phi_{m}\right)$-fields to preserve ordering by absolute discriminants is, roughly speaking, measured by the length of $[\underline{\alpha}, \underline{\omega}]$. For $\phi_{n}$ and $\phi_{m}$ coming from faithful transitive permutation representations, a very common situation is $\underline{\alpha} \leq 1 \leq \underline{\omega}$. This tendency gets stronger as $n$ and $m$ increase to $|G|$. For example, for $\left(A_{5}, \phi_{20}, \phi_{30}\right)$ the partition matrix is

$$
\left(\begin{array}{ccc}
2^{10} & 3^{6} 1^{2} & 5^{4} \\
2^{14} 1^{2} & 3^{10} & 5^{6}
\end{array}\right)
$$

and the comparison interval works out to $\left[\frac{9}{10}, \frac{15}{14}\right] \approx[0.90,1.07]$. 
7C. The right tame-wild principle often holds for $\left(G, \phi_{i}, \phi_{r}\right)$. Applying (7-3) in our setting gives

$$
\mathfrak{d}_{K / F} \mid \mathfrak{d}_{K \text { gal } / F}
$$

when $K$ is a field. This relation holds also when $K$ is an algebra, as can be seen by expressing $K$ as a product of fields and comparing each factor to the field $K^{\mathrm{gal}}$.

The critical quantity is simply expressed as

$$
\underline{\omega}\left(G, \phi_{i}, \phi_{r}\right)=\max _{\tau \in G^{\sharp 0}} \frac{\underline{c}_{\tau}\left(\phi_{i}\right)}{\underline{c}_{\tau}\left(\phi_{r}\right)} .
$$

The denominator depends only on the order $\bar{\tau}$ of $\tau$ via $\underline{c}_{\tau}\left(\phi_{r}\right)=(\bar{\tau}-1) / \bar{\tau}$. For the more complicated numerator, one has $\underline{c}_{\tau}\left(\phi_{i}\right) \leq(\bar{\tau}-1) / \bar{\tau}$, with equality if and only if the partition $\lambda_{\tau}\left(\phi_{i}\right)$ has the form $\bar{\tau}^{n / \bar{\tau}}=\bar{\tau} \ldots \bar{\tau}$. A permutation is semiregular if all cycles have the same length. Therefore $\underline{\omega}\left(G, \phi_{r}, \phi_{i}\right) \leq 1$, with equality if and only if $G$ contains a nonidentity element which is semiregular. Summarizing:

Proposition 7.1. Let $G \subseteq S_{n}$ be a permutation group containing a nonidentity semiregular element, $\phi_{i}$ the given permutation character, and $\phi_{r}$ the regular character. Then the right tame-wild principle holds for $\left(G, \phi_{i}, \phi_{r}\right)$ with

$$
\underline{\omega}\left(G, \phi_{i}, \phi_{r}\right)=1 .
$$

However, this principle is nothing more than the classical statement that for any $\left(K, K^{\mathrm{gal}}\right)$ of type $\left(G, \phi_{i}, \phi_{r}\right)$, one has $\mathfrak{d}_{K / F} \mid \mathfrak{d}_{K}$ gal $/ F$.

7D. Elusive groups. In the global setting, we are mainly interested in the case when $K$ is a field and thus $G$ is transitive. A transitive permutation group which does not contain a nonidentity semiregular element is called an elusive group [Cameron et al. 2002]. So Proposition 7.1 is the best statement for nonelusive transitive groups, but the situation needs to be investigated further for elusive groups.

Elusive groups are aptly named in that they are relatively rare. The smallest $n$ for which $S_{n}$ contains an elusive group is $n=12$. There are five elusive groups in $S_{12}$ up to permutation equivalence, listed in Table 1, all subgroups of the Mathieu group $M_{11}$ in its transitive degree twelve realization 12T272. Here and in the sequel we use the T-notation for transitive permutation groups introduced in [Conway et al. 1998] and available online in several places, including [LMFDB 2013].

The following proposition treats these five groups.

Proposition 7.2. The right tame-wild principle for $\left(G, \phi_{i}, \phi_{r}\right)$ holds for the elusive groups $12 \mathrm{~T} 46,12 \mathrm{~T} 84,12 \mathrm{~T} 181$, and $12 \mathrm{~T} 272$ with $\underline{\omega}\left(G, \phi_{i}, \phi_{r}\right)=\frac{20}{21}$. Thus the strengthening $\mathfrak{d}_{K / F} \mid \mathfrak{d}_{K^{\text {gal }} / F}^{20 / 21}$ of (7-5) holds for these groups. For $12 \mathrm{~T} 47$, one has $\underline{\omega}\left(12 \mathrm{~T} 47, \phi_{i}, \phi_{r}\right)=\frac{8}{9}$. Extensions $\left(K_{i}, K_{r}\right)$ from (7-6) give an counterexample to the tame-wild principle over $\mathbb{Q}(\sqrt{-3})$, but there is no counterexample over $\mathbb{Q}$. 


\begin{tabular}{c|ccccccc|cc}
$12 \mathrm{~T} 46$ & $\cong C_{3}^{2}: Q_{8}$ & $\sqrt{ }$ & $\sqrt{ }$ & $\sqrt{ }$ & & & $\sqrt{ }$ & & \\
$12 \mathrm{~T} 47$ & $\cong M_{9}$ & $\sqrt{ }$ & $\sqrt{ }$ & $\sqrt{ }$ & & & & & \\
$12 \mathrm{~T} 84 \cong C_{3}^{2}: \hat{Q}_{8}$ & $\sqrt{ }$ & $\sqrt{ }$ & $\sqrt{ }$ & & $\sqrt{ }$ & $\sqrt{ }$ & & & \\
$12 \mathrm{~T} 181 \cong M_{10}$ & $\sqrt{ }$ & $\sqrt{ }$ & $\sqrt{ }$ & $\sqrt{ }$ & & $\sqrt{ }$ & & & \\
$12 \mathrm{~T} 272 \cong M_{11}$ & $\sqrt{ }$ & $\sqrt{ }$ & $\sqrt{ }$ & $\sqrt{ }$ & $\sqrt{ }$ & $\sqrt{ }$ & $\sqrt{ }$ & & \\
\hline & $2 \mathrm{~A}$ & $3 \mathrm{~A}$ & $4 \mathrm{~A}$ & $5 \mathrm{~A}$ & $6 \mathrm{~A}$ & $8 \mathrm{~A}$ & $11 \mathrm{~A}$ & $Q_{8}$ & $I$ \\
$\lambda_{\tau}\left(\phi_{i}\right)$ & $2^{4} 1^{4}$ & $3^{3} 1^{3}$ & $4^{2} 2^{2}$ & $5^{2} 1^{2}$ & 6321 & 84 & $(11) 1$ & 84 & \\
$c_{\tau}\left(\phi_{i}\right)$ & 4 & 6 & 8 & 8 & 8 & 10 & 10 & 10 & $\leq 10$ \\
$\underline{c}_{\tau}\left(\phi_{r}\right)$ & $1 / 2$ & $2 / 3$ & $3 / 4$ & $4 / 5$ & $5 / 6$ & $7 / 8$ & $10 / 11$ & $7 / 8$ & $(|I|-1) /|I|$ \\
$\underline{c}_{\tau}^{\prime}$ & $2 / 3$ & $3 / 4$ & $8 / 9$ & $5 / 6$ & $4 / 5$ & $20 / 21$ & $11 / 12$ & $20 / 21$ & $\leq 20 / 21$
\end{tabular}

Table 1. Information used in the proof of Proposition 7.2.

Proof. The part below the line of Table 1 supports applying the broad and inertial methods for $12 \mathrm{~T} 272 \cong M_{11}$. Thus the line labeled $\tau$ lists out the seven elements of $12 \mathrm{~T} 272^{\sharp 0}$. The next two lines gives the corresponding dodecic partitions $\lambda_{\tau}\left(\phi_{i}\right)$ and conductors $c_{\tau}\left(\phi_{i}\right)=12 \underline{c}_{\tau}\left(\phi_{i}\right)$ respectively. The next lines give

$$
\underline{c}_{\tau}\left(\phi_{r}\right)=\frac{c_{\tau}\left(\phi_{r}\right)}{\left|M_{11}\right|} \quad \text { and } \quad \underline{c}_{\tau}^{\prime}=\frac{\underline{c}_{\tau}\left(\phi_{i}\right)}{\underline{c}_{\tau}\left(\phi_{r}\right)}
$$

Thus the comparison interval is $[\underline{\alpha}, \underline{\omega}]=\left[\frac{2}{3}, \frac{20}{21}\right]$.

One inertial subgroup of $12 \mathrm{~T} 272$ is $Q_{8}$, which has orbit partition 84. As indicated in the second-to-last column of Table 1 , its associated quantity is $\underline{c}_{Q_{8}}^{\prime}=\frac{20}{21}$, which is the right endpoint of $\left[\frac{2}{3}, \frac{20}{21}\right]$. In general, the difficulty with the inertial method is that there can be many inertial N-subgroups $I$ to inspect. However here we can treat them all at once as follows. Since none of the elusive groups from the complete list are themselves inertial groups, $I$ must act intransitively and so $c_{I}\left(\phi_{i}\right) \leq 10$. Also $\underline{c}_{I}\left(\phi_{r}\right)=(|I|-1) /|I| \geq \frac{7}{8}$, since $\mathrm{N}$-groups have order at least 8 . So, as indicated by the table, $\underline{c}_{I}^{\prime} \leq \frac{20}{21}$. Thus our initial case $Q_{8}$ was in fact the worst case, and the right tame-wild principle holds for $\left(M_{11}, \phi_{i}, \phi_{r}\right)$.

The part of the table above the line gives the partitions which arise for all the $G$, as a subset of those that we have listed for 11T272. The smaller groups 12T46, $12 \mathrm{~T} 84$, and $12 \mathrm{~T} 181$ still have elements of cycle type 84 , and so the same argument goes through for them, proving the tame-wild principle for $\left(G, \phi_{i}, \phi_{r}\right)$ in these cases. Note that our uniform treatment of all the $G$ uses that $\underline{c}_{\tau}\left(\phi_{r}\right)$ is independent of $G$; in contrast, the unnormalized quantity $c_{\tau}\left(\phi_{r}\right)$ depends on $G$.

A counterexample seems likely for $G=12 \mathrm{~T} 47$ because it contains $Q_{8}$ and its comparison interval is only $[\underline{\alpha}, \underline{\omega}]=\left[\frac{2}{3}, \frac{8}{9}\right]$, with $\frac{8}{9}$ being considerably less than $\frac{20}{21}$. However, as discussed in reference to (6-2), there are no quaternionic extensions 
of $\mathbb{Q}_{2}$ giving counterexamples to the universal tame-wild principle for $Q_{8}$. Other candidate $I$ do not work either, and we are forced to leave $\mathbb{Q}$ as a ground field.

Our counterexample comes from fields $K_{n}=\mathbb{Q}[x] / f_{n}(x)$ with discriminants $D_{n} \in \mathbb{Z}$ and Galois groups $G_{n}=\operatorname{Gal}\left(K_{n}^{\mathrm{gal}} / \mathbb{Q}\right)$ as follows:

\begin{tabular}{r|lcc}
$n$ & $f_{n}(x)$ & $D_{n}$ & $G_{n}$ \\
\hline 8 & $x^{8}+6 x^{4}-3$ & $-2^{16} 3^{7}$ & $\hat{Q}_{8}$ \\
9 & $x^{9}-3 x^{8}+18 x^{5}+18 x^{4}-27 x+9$ & $-2^{16} 3^{15}$ & $9 \mathrm{~T} 19$ \\
12 & $x^{12}-6 x^{10}-4 x^{9}+12 x^{7}-36 x^{5}+30 x^{4}+8 x^{3}-8$ & $2^{22} 3^{18}$ & $12 \mathrm{~T} 84$
\end{tabular}

The overgroup $12 \mathrm{~T} 84 \cong C_{3}^{2}: \hat{Q}_{8} \supset 12 \mathrm{~T} 47$ was chosen because it contains not just $Q_{8}$ but also $\hat{Q}_{8}$. The nonic group 9T19 is a lower degree realization of $12 \mathrm{~T} 84$, where the isomorphism with $C_{3}^{2}: \hat{Q}_{8}$ is naturally realized. The field $K_{8}$ was chosen as a strong candidate from which to build a counterexample, because $\operatorname{Gal}\left(K_{8}^{\mathrm{gal}} / \mathbb{Q}\right)$ is its own decomposition group with slope content $[2,2,2.5]^{2}$ as in (6-2). The field $K_{9}$ was extracted from the database [Jones and Roberts $\geq 2014$ ] as a 9T19 field with $K_{8}$ as a resolvent, and then $K_{12}$ was obtained from $K_{9}$ by resolvent calculations.

The splitting field $K_{12}^{\mathrm{gal}}$ contains $\mathbb{Q}(\sqrt{-3})$ with $\operatorname{Gal}\left(K_{12}^{\mathrm{gal}} / \mathbb{Q}(\sqrt{-3})\right)=12 \mathrm{~T} 47$ by construction. The root discriminant of $K_{12}^{\text {gal }}$ is $2^{2} 3^{127 / 72}$, as computed by using the website of [Jones and Roberts 2006] to analyze ramification in $K_{9} / \mathbb{Q}$. Here the exponent 2 can be confirmed from a standard computation associated with the slope content $[2,2,2.5]^{2}$, namely $\frac{2}{8}+\frac{2}{4}+\frac{2.5}{2}=2$. On the other hand $K_{12}$ has root discriminant $2^{22 / 12} 3^{18 / 12}$. The quotient $\frac{22}{12} / 2=\frac{11}{12}$ is to the right of the root-normalized tame hull $[\underline{\alpha}, \underline{\omega}]=\left[\frac{2}{3}, \frac{8}{9}\right]$, giving a counterexample to the right tame-wild principle for $\left(12 \mathrm{~T} 47, \phi_{i}, \phi_{r}\right)$ over $\mathbb{Q}(\sqrt{-3})$.

7E. The left tame-wild principle always holds for $\left(G, \phi_{i}, \phi_{r}\right)$. The following theorem shows that an important part of the tame-wild principle holds for all finite groups $G$.

Theorem 7.3. Let $G \subseteq S_{n}$ be any permutation group, $\phi_{i}$ the given permutation character, and $\phi_{r}$ the regular character. Let $\mathscr{F}(G)$ be the maximal number of fixed points of a nonidentity element of $G$. Then the left tame-wild principle holds for $\left(G, \phi_{i}, \phi_{r}\right)$ with

$$
\underline{\alpha}\left(G, \phi_{i}, \phi_{r}\right)=1-\frac{\mathscr{F}(G)}{n} .
$$

Thus for any $\left(K, K^{\mathrm{gal}}\right)$ of type $\left(G, \phi_{i}, \phi_{r}\right)$, one has $\mathfrak{d}_{K^{\mathrm{gal}} / F}^{1-\mathscr{F}(G) / n} \mid \mathfrak{d}_{K / F}$.

Proof. We apply the broad method. Let $\tau$ be an arbitrary element of $G^{\sharp 0}$ and call its order $t$. Consider the corresponding column $\left(\begin{array}{l}\lambda \\ \Lambda\end{array}\right)$ of the partition matrix $P\left(G, \phi_{i}, \phi_{r}\right)$. Then $\lambda=\prod_{k=1}^{t} k^{m_{k}}$ is some partition of $n$ and $\Lambda=t^{|G| / t}$ is the corresponding partition of $|G|$. 
The projective matrices $T^{\prime}\left(G, \phi_{i}, \phi_{r}\right)$ and $\hat{T}^{\prime}\left(G, \phi_{i}, \phi_{r}\right)$ have just one row each. The entries in the $\tau$ column are respectively $c(\lambda) / c(\Lambda)$ and $\hat{c}(\lambda) / \hat{c}(\Lambda)$. Their difference is positive:

$$
\begin{aligned}
\frac{\hat{c}(\lambda)}{\hat{c}(\Lambda)}-\frac{c(\lambda)}{c(\Lambda)} & =\frac{\sum_{k=2}^{t} m_{k} k}{|G|}-\frac{\sum_{k=2}^{t} m_{k}(k-1)}{|G|(t-1) / t} \\
& =\frac{1}{|G|} \sum_{k=2}^{t} m_{k} \frac{k(t-1)}{t-1}-\frac{1}{|G|} \sum_{k=2}^{t} m_{k} \frac{t(k-1)}{t-1} \\
& =\frac{1}{|G|} \sum_{k=2}^{t} m_{k} \frac{(k t-k)-(k t-t)}{t-1} \\
& =\frac{1}{|G|} \sum_{k=2}^{t} m_{k} \frac{t-k}{t-1} \geq 0 .
\end{aligned}
$$

Thus $\hat{c}(\lambda) / \hat{c}(\Lambda) \geq c(\lambda) / c(\Lambda)$, and so certainly all the $\hat{c}(\lambda) / \hat{c}(\Lambda)$ are at least

$$
\alpha\left(G, \phi_{i}, \phi_{r}\right)=\min _{\tau \in G^{\sharp 0}} \frac{c_{\tau}(\lambda)}{c_{\tau}(\Lambda)} .
$$

Thus the left tame-wild principle holds for $\left(G, \phi_{i}, \phi_{r}\right)$, and moreover we can compute $\alpha\left(G, \phi_{i}, \phi_{r}\right)$ using $\hat{c}_{\tau}$ rather than $c_{\tau}$, giving

$$
\alpha\left(G, \phi_{i}, \phi_{r}\right)=\min _{\tau \in G^{\sharp 0}} \frac{\hat{c}_{\tau}(\lambda)}{\hat{c}_{\tau}(\Lambda)}=\min _{\tau \in G^{\sharp 0}} \frac{n-m_{1}}{|G|}=\frac{n-\mathscr{F}(G)}{|G|} .
$$

Switching to the mean-root normalization gives $\underline{\alpha}\left(G, \phi_{i}, \phi_{r}\right)=1-\mathscr{F}(G) / n$.

Number field tabulation. For certain solvable transitive groups $G \subset S_{n}$, the techniques of [Jones and Wallington 2012] let one compute all degree $n$ fields $K$ of type $G$ where $\mid \mathfrak{d}_{K}$ gal $/ \mathbb{Q} \mid$ is at most some constant $\beta$. Then the theorem just proved can be applied through its corollary $\left|\mathfrak{d}_{K^{\mathrm{gal}} / \mathbb{Q}}^{1-\mathscr{F}(G) / n}\right| \leq\left|\mathfrak{d}_{K / \mathbb{Q}}\right|$ to obtain all $K$ with $\left|\mathfrak{d}_{K / \mathbb{Q}}\right|$ at most $B=\beta^{1-\mathscr{F}(G) / n}$. This computation is carried out in [Jones 2013] for the primitive nonic groups 9T9, 9T14, 9T15, 9T16, 9T23, and 9T27 to obtain the corresponding nonic fields with smallest absolute discriminant. This particular application served as the catalyst for the present paper.

\section{Examples and counterexamples}

The positive and negative results of the previous sections give one a good idea of the extent to which the tame-wild principle holds and how it can be applied. We now refine this picture, by considering various $\left(G, \phi_{1}, \ldots, \phi_{r}\right)$ of interest and determining whether the tame-wild principle holds. In Section $8 \mathrm{~A}$, we give examples illustrating the broad method and the inertial method. In Section 8B, we conclude 
by arguing that counterexamples to the tame-wild principle from pairs $\left(K_{1}, K_{2}\right) / \mathbb{Q}$ of number fields are not easily found, but present one such counterexample with Galois group 12T112 of order 192.

8A. The broad and inertial methods. We illustrate the two methods of Section 4B with positive results for three $\mathrm{N}$-groups.

The broad method for $\left(\mathrm{Aff}_{3}\left(\mathbb{F}_{2}\right), \phi_{7}, \phi_{8}, \phi_{8 a}, \phi_{8 b}\right)$. The group $\mathrm{Aff}_{3}\left(\mathbb{F}_{2}\right)$ provides a simple illustration of the broad method in the setting $r=4$. It has five nontrivial small permutation representations $\rho_{7 a}, \rho_{7 b}, \rho_{8}, \rho_{8 a}, \rho_{8 b}$, with images the permutation groups 7T5, 7T5, 8T37, 8T48, 8T48. The first three representations are through the quotient $\mathrm{GL}_{3}\left(\mathbb{F}_{2}\right) \cong \mathrm{PGL}_{2}\left(\mathbb{F}_{7}\right)$ while the last two are faithful. The representations $\rho_{7 a}$ and $\rho_{7 b}$ share a common character $\phi_{7}$. They are thus arithmetically equivalent and we call them identical twins. The representations $\rho_{8 a}$ and $\rho_{8 b}$ have different characters $\phi_{8 a}$ and $\phi_{8 b}$ and so we call them fraternal twins. The four characters $\phi_{7}$, $\phi_{8}, \phi_{8 a}$, and $\phi_{8 b}$ are linearly independent.

Figure 3 first presents the partition matrix $P=P\left(\mathrm{Aff}_{3}\left(\mathbb{F}_{2}\right), \phi_{7}, \phi_{8}, \phi_{8 a}, \phi_{8 b}\right)$ and the broad and tame matrices derived from it. For visualization purposes, it then drops consideration of $\phi_{8}$. After this projection, it plots the columns of $\hat{T}^{\prime}$ as $+\mathrm{s}$ and those of $T^{\prime}$ as $\bullet$ s. Since the $+\mathrm{s}$ are in the hull $T_{+}^{\prime}$ of the $\bullet \mathrm{s}$, the tame-wild principle holds for $\left(\operatorname{Aff}_{3}\left(\mathbb{F}_{2}\right), \phi_{7}, \phi_{8 a}, \phi_{8 b}\right)$. Working more algebraically, as described in Section $4 \mathrm{C}$, one can verify the analogous convexity assertion in the presence of $\phi_{8}$, giving the first sentence of the following result.

Proposition 8.1. The tame-wild principle holds for $\left(\mathrm{Aff}_{3}\left(\mathbb{F}_{2}\right), \phi_{7}, \phi_{8}, \phi_{8 a}, \phi_{8 b}\right)$. In particular, to find all 8T48 extensions with $\left|\mathfrak{D}_{K_{8 a} / F}\right| \leq B$, one need look only at 7T5 extensions with $\left|\mathfrak{D}_{K_{7} / F}\right| \leq B$ and select from among the octic resolvents of their 14T34 quadratic overfields.

The second sentence comes from an understanding of the algebraic meaning of Figure 3. Associate variables $u, a$, and $b$ to $\phi_{7}, \phi_{8 a}$, and $\phi_{8 b}$ respectively. The four sides of the trapezoid $T_{+}^{\prime}\left(\mathrm{Aff}_{3}\left(\mathbb{F}_{2}\right), \phi_{7}, \phi_{8 a}, \phi_{8 b}\right)$ in the drawn $(u / b, a / b)$ plane correspond to the four faces of the cone $T_{+}\left(\mathrm{Aff}_{3}\left(\mathbb{F}_{2}\right), \phi_{7}, \phi_{8 a}, \phi_{8 b}\right)$ in $(u, a, b)$ space. These four faces correspond to the four inequalities on local exponents on the left and they translate into divisibility relations among either local or global discriminants on the right:

$$
\begin{aligned}
& u \leq a \leq u+b, \quad \mathfrak{D}_{K_{7} / F}\left|\mathfrak{D}_{K_{8 a} / F}\right| \mathfrak{D}_{K_{7} / F} \mathfrak{D}_{K_{8 b} / F}, \\
& u \leq b \leq u+a, \quad \mathfrak{D}_{K_{7} / F}\left|\mathfrak{D}_{K_{8 b} / F}\right| \mathfrak{D}_{K_{7} / F} \mathfrak{D}_{K_{8 a} / F} .
\end{aligned}
$$

For tabulations of all extensions $K_{8 a} / F$ with $\left|\mathfrak{D}_{K_{8 a} / F}\right|$ at most some bound $B$, the procedure referred to by the proposition is to look for all $K_{7} / F$ with $\left|\mathfrak{D}_{K_{7} / F}\right| \leq B$, 


\begin{tabular}{c|ccccccccc}
$\tau$ & $2 \mathrm{~A}$ & $2 \mathrm{~B}$ & $2 \mathrm{C}$ & $3 \mathrm{~A}$ & $4 \mathrm{~A}$ & $4 \mathrm{~B}$ & $4 \mathrm{C}$ & $6 \mathrm{~A}$ & $7 \mathrm{~A}$ \\
\hline $\boldsymbol{5}$ & $1^{7}$ & 22111 & 22111 & 331 & 22111 & 421 & 421 & 331 & 7 \\
$1^{8}$ & 2222 & 2222 & 3311 & 2222 & 44 & 44 & 3311 & 71 \\
2222 & 221111 & 2222 & 3311 & 44 & 4211 & 44 & 62 & 71 \\
& 2222 & 2222 & 221111 & 3311 & 44 & 44 & 4211 & 62 & 71 \\
\hline$T$ & 0 & 4 & 4 & 6 & 4 & 6 & 6 & 6 & 7 \\
+ & 0 & 8 & 8 & 6 & 8 & 8 & 8 & 6 & 7 \\
& 8 & 4 & 8 & 6 & 8 & 6 & 8 & 8 & 7 \\
& 8 & 8 & 4 & 6 & 8 & 8 & 6 & 8 & 7 \\
\hline & 0 & 2 & 2 & 4 & 2 & 4 & 4 & 4 & 6 \\
& 0 & 4 & 4 & 4 & 4 & 6 & 6 & 4 & 6 \\
& 4 & 2 & 4 & 4 & 6 & 4 & 6 & 6 & 6 \\
& 4 & 4 & 2 & 4 & 6 & 6 & 4 & 6 & 6
\end{tabular}

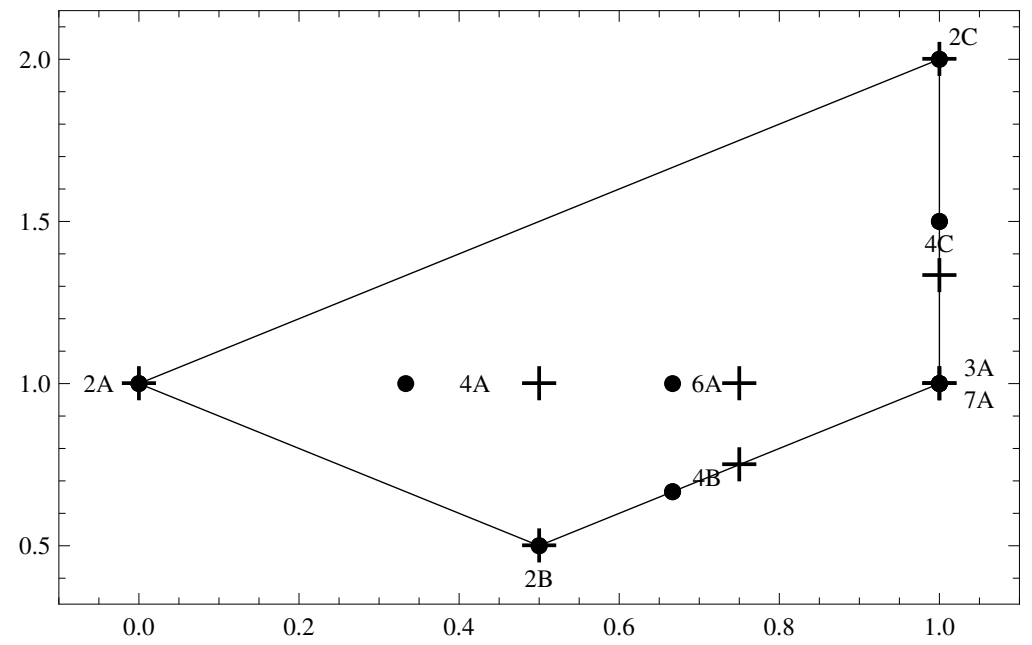

Figure 3. Top: The partition matrix, broad matrix, and tame matrix for $\left(\operatorname{Aff}_{3}\left(\mathbb{F}_{2}\right), \phi_{7}, \phi_{8}, \phi_{8 a}, \phi_{8 b}\right)$. Bottom: the broad hull and tame hulls coinciding after removing $\phi_{8}$ from consideration, proving the tame-wild principle for $\left(\mathrm{Aff}_{3}\left(\mathbb{F}_{2}\right), \phi_{7}, \phi_{8 a}, \phi_{8 b}\right)$.

take suitable square roots to pass from $7 \mathrm{~T} 5$ fields to $14 \mathrm{~T} 34$ fields, and then use resolvents to obtain the desired $8 \mathrm{~T} 48$ fields.

The inertial method for $\left(S_{6}, \phi_{6 a}, \phi_{6 b}, \phi_{10}\right)$. The group $S_{6}$ has three faithful permutation representations of degree at most ten: two sextic ones $\rho_{6 a}$ and $\rho_{6 b}$ interchanged by the outer automorphism of $S_{6}$, and a decic one $\rho_{10}$ coming from the exceptional isomorphism $S_{6} \cong \mathrm{PSL}_{2}\left(\mathbb{F}_{9}\right) \cdot \mathrm{Gal}\left(\mathbb{F}_{9} / \mathbb{F}_{3}\right)=10 \mathrm{~T} 32 \subset S_{10}$. 


\begin{tabular}{c|cccccccccc} 
& $2 \mathrm{~A}$ & $2 \mathrm{~B}$ & $2 \mathrm{C}$ & $3 \mathrm{~A}$ & $3 \mathrm{~B}$ & $4 \mathrm{~A}$ & $4 \mathrm{~B}$ & $5 \mathrm{~A}$ & $6 \mathrm{~A}$ & $6 \mathrm{~B}$ \\
\hline$P$ & 222 & $21^{4}$ & 2211 & 33 & 3111 & 42 & 411 & 51 & 6 & 321 \\
& $21^{4}$ & 222 & 2211 & 3111 & 33 & 42 & 411 & 51 & 321 & 6 \\
& $2^{3} 1^{4}$ & $2^{3} 1^{4}$ & $2^{4} 1^{2}$ & 3331 & 3331 & 4411 & 442 & 55 & 631 & 631 \\
\hline$\hat{T}$ & 6 & 2 & 4 & 6 & 3 & 6 & 4 & 5 & 6 & 5 \\
+ & 2 & 6 & 4 & 3 & 6 & 6 & 4 & 5 & 5 & 6 \\
& 6 & 6 & 8 & 9 & 9 & 8 & 10 & 10 & 9 & 9 \\
\hline$T$ & 3 & 1 & 2 & 4 & 2 & 4 & 3 & 4 & 5 & 3 \\
$\bullet$ & 1 & 3 & 2 & 2 & 4 & 4 & 3 & 4 & 3 & 5 \\
& 3 & 3 & 4 & 6 & 6 & 6 & 7 & 8 & 7 & 7
\end{tabular}

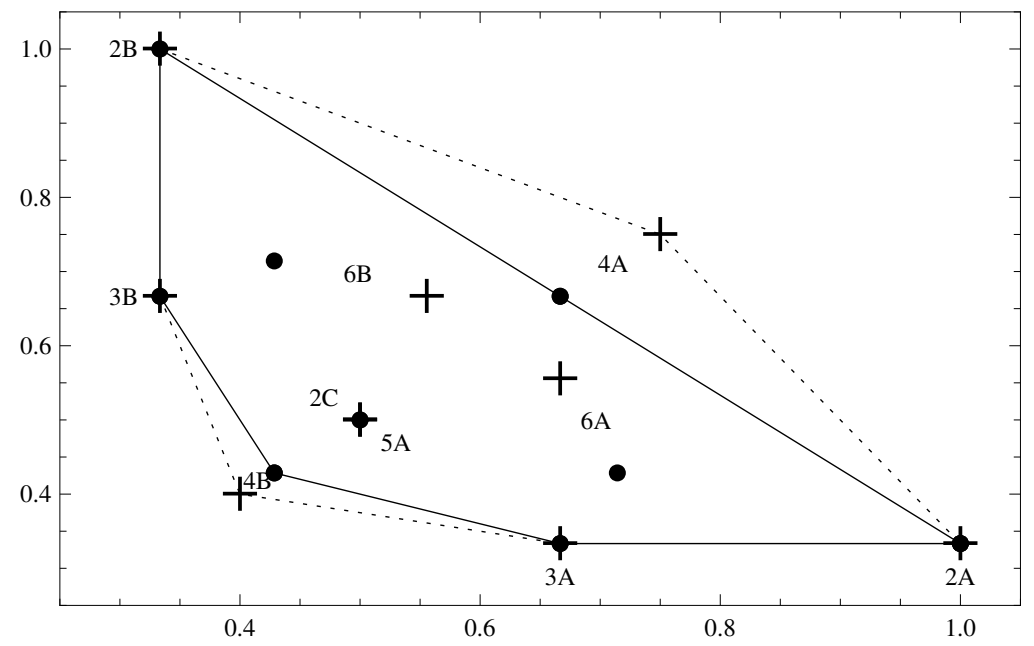

Figure 4. Top: The partition matrix, broad matrix, and tame matrix for $\left(S_{6}, \phi_{6 a}, \phi_{6 b}, \phi_{10}\right)$. Bottom: the broad hull strictly containing the tame hull, showing that the broad method does not suffice to prove the tame-wild principle for $\left(S_{6}, \phi_{6 a}, \phi_{6 b}, \phi_{10}\right)$.

Figure 4 presents our standard analysis of the situation. Since some $+\mathrm{s}$ are outside of the tame hull $T_{+}^{\prime}\left(S_{6}, \phi_{6 a}, \phi_{6 b}, \phi_{10}\right)$, the broad method does not suffice for $\left(S_{6}, \phi_{6 a}, \phi_{6 b}, \phi_{10}\right)$. However after projection to the horizontal axis, the $+\mathrm{s}$ are indeed in the convex hull of the $\bullet \mathrm{s}$, so that the broad method establishes the tamewild principle for $\left(S_{6}, \phi_{6 a}, \phi_{10}\right)$. Also the ratios $a / b$ for the + points $(a, b)$ are within the interval $\left[\frac{1}{3}, 3\right]$ formed by the ratios for the $\bullet$ points, proving the tame-wild principle for $\left(S_{6}, \phi_{6 a}, \phi_{6 b}\right)$.

In fact, the tame-wild principle is true for $\left(S_{6}, \phi_{6 a}, \phi_{6 b}, \phi_{10}\right)$ as follows. The only inertial subgroups not covered by previous considerations are $I_{1}=D_{4} \times C_{2}$ 
and the twin pair $\left(I_{2}, I_{3}\right)=\left(A_{4} \times C_{2}, 6 \mathrm{~T} 6\right)$. The orbit partitions in the three cases are $(42,42,442),(42,6,64)$, and $(6,42,64)$. The associated conductor vectors are then $(4,4,7),(4,5,8)$, and $(5,4,8)$. Their projectivized versions are $\left(\frac{4}{7}, \frac{4}{7}\right),\left(\frac{1}{2}, \frac{5}{8}\right)$, and $\left(\frac{5}{8}, \frac{1}{2}\right)$. Since these points are visibly in $T_{+}^{\prime}\left(S_{6}, \phi_{6 a}, \phi_{6 b}, \phi_{10}\right)$, the tame-wild principle holds. We have given this argument to illustrate how the inertial method typically applies. However in this case the inertial groups $I_{2}$ and $I_{3}$ could also have been treated by using the techniques from Section 6, as in fact the universal tame-wild principle holds for $A_{4} \times C_{2}$.

Summarizing, we have proved the first sentence:

Proposition 8.2. The tame-wild principle holds for $\left(S_{6}, \phi_{6 a}, \phi_{6 b}, \phi_{10}\right)$. In particular to find all decic $S_{6}$-extensions with $\left|\mathfrak{D}_{K_{10} / F}\right| \leq B$, one need only look at sextic $S_{6}$-extensions with $\left|\mathfrak{D}_{K_{6 a} / F}\right| \leq B^{2 / 3}$ and select from among their decic resolvents.

For the second sentence, note first that the locations of the rightmost and highest points of the tame hull in Figure 4 respectively correspond to the equivalent statements $\mathfrak{D}_{K_{6 a} / F} \mid \mathfrak{D}_{K_{10} / F}$ and $\mathfrak{D}_{K_{6 b} / F} \mid \mathfrak{D}_{K_{10} / F}$. Each of these says that to find all decics with absolute discriminant $\leq B$, it suffices to look at all sextics up to that bound. A considerable improvement is to see that the long diagonal boundary between them corresponds to $\mathfrak{D}_{K_{6 a} / F} \mathfrak{D}_{K_{6 b} / F} \mid \mathfrak{D}_{K_{10} / F}^{4 / 3}$ which implies the statement. The broad method for $\left(W\left(E_{6}\right), \phi_{27}, \phi_{36}, \phi_{40 a}, \phi_{40 b}, \phi_{45}\right)$. As we have seen in Section $7 \mathrm{E}$ and by the earlier examples of this subsection, the broad method works well in the setting $r=2$. As $r$ increases, the difference between $a_{\tau}$ and $\hat{a}_{\tau}$ becomes more visible, and the broad method often fails even when the tame-wild principle is true, as we just saw for $\left(S_{6}, \phi_{6 a}, \phi_{6 b}, \phi_{10}\right)$.

A clear illustration of the effectiveness of the broad method and its decay with increasing $r$ comes from the Weyl group $W\left(E_{6}\right)$ of order $51840=2^{6} 3^{4} 5$ and the permutation characters $\phi_{27}, \phi_{36}, \phi_{40 a}, \phi_{40 b}, \phi_{45}$ corresponding to five maximal subgroups [Conway et al. 1985]. The broad method immediately shows that the tame-wild principle for $\left(W\left(E_{6}\right), \phi_{u}, \phi_{v}\right)$ holds for all ten possibilities for $\{u, v\}$. From ten pictures like Figures 3 and 4, now quite involved since $\left|W\left(E_{6}\right)^{\sharp 0}\right|=24$, the broad method establishes the tame-wild principle in exactly four of the ten cases $\left(W\left(E_{6}\right), \phi_{u}, \phi_{v}, \phi_{w}\right)$ as follows.

Proposition 8.3. For $\{u, v, w\}=\{27,36,40 a\},\{27,40 a, 40 b\},\{36,40 a, 40 b\}$, and $\{36,40 b, 45\}$, the tame-wild principle holds for $\left(W\left(E_{6}\right), \phi_{u}, \phi_{v}, \phi_{w}\right)$.

Pursuing this situation further with the inertial method would be harder, because $W\left(E_{6}\right)$ has many 2-inertial and 3-inertial subgroups.

8B. Best counterexamples. Let $G$ be a group for which the universal tame-wild principle fails. Then there exists a vector $v \in \mathbb{Q}\left(G^{\sharp}\right)$ for which the tame-wild principle fails for $(G,\langle v\rangle)$. There are infinitely many solutions to $\phi_{1}-\phi_{2} \in\langle v\rangle$ 
with the $\phi_{i}$ permutation characters. So any failure of the universal tame-wild principle can be converted to a failure in the setting $\left(G, \phi_{1}, \phi_{2}\right)$ of the introduction. By switching $\phi_{1}$ and $\phi_{2}$ if necessary, it can be converted to a failure of the left tame-wild principle for $\left(G, \phi_{1}, \phi_{2}\right)$.

However these counterexamples are not guaranteed to have immediate bearing on our applications to tabulating number fields. All that is asserted by the failure of the principle for $\left(G, \phi_{1}, \phi_{2}\right)$ is that there exists a pair of local extensions $\left(K_{1}, K_{2}\right) / F$ of the given type with

$$
\mathfrak{D}_{K_{2} / F}^{\alpha\left(G, \phi_{1}, \phi_{2}\right)} \mid \mathfrak{D}_{K_{1} / F}
$$

not holding. More directly relevant would be global counterexamples with the $\phi_{i}$ both coming from faithful transitive permutation representations and the extensions $K_{i} / F$ full in the sense of each having Galois group $\operatorname{Gal}\left(K^{\mathrm{gal}} / F\right)$ all of $G$. More demanding still is to ask for counterexamples of this sort with $F=\mathbb{Q}$. Finally, one can seek examples for which even the weaker numerical statement

$$
\left|\mathfrak{D}_{K_{2} / \mathbb{Q}}\right|^{\alpha\left(G, \phi_{1}, \phi_{2}\right)} \leq\left|\mathfrak{D}_{K_{1} / \mathbb{Q}}\right|
$$

fails. Examples of this explicit nature often do not exist for a given $G$, and even when they exist they can be hard to find. The rest of this subsection discusses the construction of global counterexamples built from one of the two local counterexamples with $I=Q_{8}$ with slope-content $\left[2,2, \frac{5}{2}\right]^{2}$ from (6-2). There are several points of contact with Section 7D, but here we find counterexamples to (8-1) over $\mathbb{Q}$.

Inadequacy of $G=\hat{Q}_{8}$ as a source of global counterexamples. The group $\hat{Q}_{8}$ itself is not a source of global counterexamples of the sort we seek because it has only two transitive faithful permutation characters and the tame-wild principle holds for the corresponding type $\left(\hat{Q}_{8}, \phi_{8}, \phi_{16}\right)$. To illustrate the best that can be done with this group, take

\begin{tabular}{c|lccc}
$n$ & $f_{n}(x)$ & $D_{n}$ & $G_{n}$ & $\left|G_{n}\right|$ \\
\hline 8 & $x^{8}+6 x^{4}-3$ & $-2^{16} 3^{7}$ & $\hat{Q}_{8}$ & 16 \\
4 & $x^{4}+6 x^{2}-3$ & $-2^{6} 3^{3}$ & $D_{4}$ & 8 \\
2 & $x^{2}+3$ & -3 & $C_{2}$ & 2
\end{tabular}

The global and 2-adic Galois groups of $f_{8}(x)$ agree, and so one has this agreement for the resolvents $f_{4}(x)$ and $f_{2}(x)$ as well. The Galois groups $G_{n}$ and the field discriminants $D_{n}$ are as indicated. The fields $K_{n}=\mathbb{Q}[x] / f_{n}(x)$ belong to transitive characters $\phi_{8}, \phi_{4}$, and $\phi_{2}$ of $\hat{Q}_{8}$.

Figure 5 is an analog of Figure 1 , but now for $\left(\hat{Q}_{8}, \phi_{8}+\phi_{2}, \phi_{8}+\phi_{4}\right)$. The algebra pair $\left(K_{8} \times K_{2}, K_{8} \times K_{4}\right) / \mathbb{Q}$ yields the exponent pair $\left(a_{2}, b_{2}\right)=(16,22)$ which is just outside the tame cone. So this pair of algebras indeed contradicts (8-1), but we are seeking counterexamples among pairs of fields. 


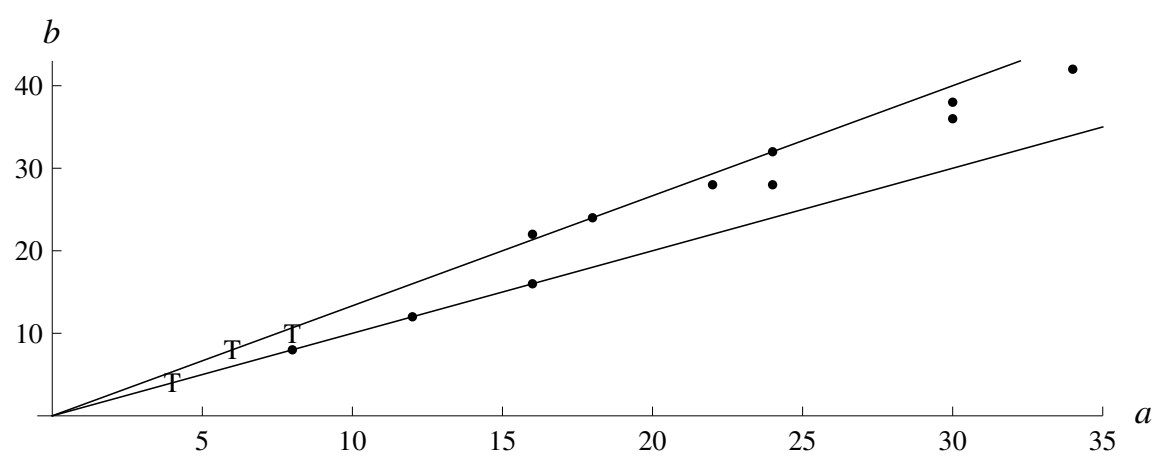

Figure 5. An analog of the introductory Figure 1 for the type $\left(\hat{Q}_{8}, \phi_{8}+\phi_{2}, \phi_{8}+\phi_{4}\right)$. The points are exactly all the possibilities for exponent pairs $\left(a_{2}, b_{2}\right)$ from wild 2-adic ramification over $\mathbb{Q}_{2}$, and $(16,22)$ is just outside the tame cone.

Failure of the inertial method for $\left(M_{12}, \phi_{12 a}, \phi_{12 b}\right)$. To get a better global counterexample corresponding to the same local counterexample, we need to replace $\hat{Q}_{8}$ by larger groups $G$ containing it. An initial key observation is that the quaternion group $Q_{8}$ is the four-point stabilizer of the Mathieu group $M_{12} \subset S_{12}$ of order $12 \cdot 11 \cdot 10 \cdot 9 \cdot 8$ in its natural action, and also one has $Q_{8} \subset \hat{Q}_{8} \subset M_{12}$. On the one hand, the given character $\phi_{12 a}$ of the Mathieu group has decomposition $\phi_{8}+\phi_{2}+2$ when restricted to $\hat{Q}_{8}$. On the other hand, there is a twin dodecic character $\phi_{12 b}$ coming from the outer involution of $M_{12}$; its restriction to $\hat{Q}_{8}$ decomposes as $\phi_{8}+\phi_{4}$.

Further group-theoretic facts are necessary for this situation to give number fields as desired. First, the partition matrix and projective tame matrix of $\left(M_{12}, \phi_{12 a}, \phi_{12 b}\right)$ are as follows:

\begin{tabular}{c|ccccccccccccc|c}
$\tau$ & $2 \mathrm{~A}$ & $2 \mathrm{~B}$ & $3 \mathrm{~A}$ & $3 \mathrm{~B}$ & $4 \mathrm{~A}$ & $4 \mathrm{~B}$ & $5 \mathrm{~A}$ & $6 \mathrm{~A}$ & $6 \mathrm{~B}$ & $8 \mathrm{~A}$ & $8 \mathrm{~B}$ & $10 \mathrm{~A}$ & $11 \mathrm{AB}$ & $Q_{8}$ \\
\hline$\lambda_{\tau}\left(\phi_{12 a}\right)$ & $2^{6}$ & $2^{4} 1^{4}$ & $3^{3} 1^{3}$ & $3^{4}$ & $4^{2} 2^{2}$ & $4^{2} 1^{4}$ & $5^{2} 1^{2} 6^{2}$ & 6321 & 84 & $821^{2}$ & $(10) 2$ & $(11) 1$ & $81^{4}$ \\
$\lambda_{\tau}\left(\phi_{12 b}\right)$ & $2^{6}$ & $2^{4} 1^{4}$ & $3^{3} 1^{3}$ & $3^{4}$ & $4^{2} 1^{4}$ & $4^{2} 2^{2}$ & $5^{2} 1^{2} 6^{2}$ & 6321 & $821^{2}$ & 84 & $(10) 2$ & $(11) 1$ & 84 \\
\hline$c_{\tau}^{\prime}$ & 1 & 1 & 1 & 1 & $\frac{8}{6}$ & $\frac{6}{8}$ & 1 & 1 & 1 & $\frac{10}{8}$ & $\frac{8}{10}$ & 1 & 1 & $\frac{7}{10}$
\end{tabular}

Thus, in the language introduced in Section $8 \mathrm{~A}$, extensions $\left(K_{12 a}, K_{12 b}\right) / F$ of full type $\left(M_{12}, \phi_{12 a}, \phi_{12 b}\right)$ are fraternal twins, this being necessary for our purposes. But they are near-identical in the sense that the interval $[\alpha, \omega]$ is small, being $\left[\frac{3}{4}, \frac{4}{3}\right]=$ $[0.75,1 . \overline{33}]$ here, rather than the intervals $\left[\frac{1}{2}, 2\right]$ and $\left[\frac{1}{3}, 3\right]$ seen in Section $8 \mathrm{~A}$ for $\operatorname{Aff}_{3}\left(\mathbb{F}_{2}\right)$-twins and $S_{6}$-twins respectively. The orbit partitions of $Q_{8}$ are as indicated above, yielding $c_{Q_{8}}\left(\phi_{12 a}\right) / c_{Q_{8}}\left(\phi_{12 b}\right)=\frac{7}{10}=0.70$ which is outside the interval $[0.75,1 . \overline{33}]$. Thus the inertial method for proving the tame-wild principle fails here. 
Failure of the tame-wild principle for $\left(M_{12}, \phi_{12 a}, \phi_{12 b}\right)$. Computing with the slopes $[2,2,2.5]^{2}$, the $8 \mathrm{~s}$ in the last column above give discriminant exponent $3 \cdot 2+4 \cdot 2.5=$ 16 while the 4 gives the discriminant exponent $3 \cdot 2=6$. So the ratio of wild conductors is $\frac{16}{22}=0 . \overline{72}$, which is still outside the interval $[0.75,1 . \overline{33}]$. Thus the tame-wild principle itself fails for $\left(M_{12}, \phi_{12 a}, \phi_{12 b}\right)$.

Smaller groups. We have looked in several places, including the two-parameter family of [Malle 2000], for twin pairs $\left(K_{12 a}, K_{12 b}\right)$ of $M_{12}$ fields with the needed quaternionic 2-adic behavior. We did not find any, and so we consider smaller groups as follows as potential sources of counterexamples:

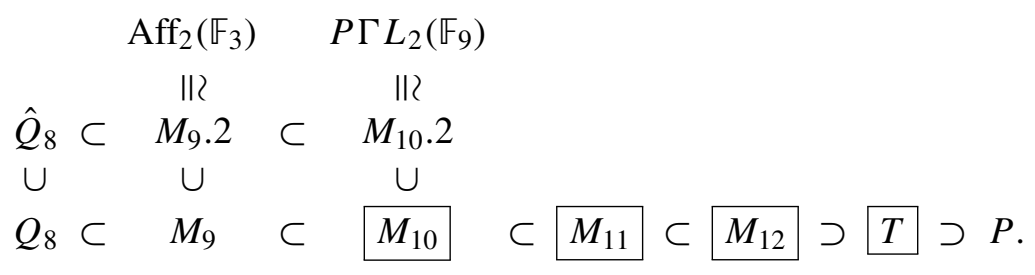

The four groups in the middle are boxed to stress that they appear in Proposition 8.4 below.

Proceeding from $M_{12}$ to the left, the groups $M_{11}$ and $M_{10}$ contain $\hat{Q}_{8}$, since $\hat{Q}_{8}$ has orbit partition 8211 . Thus, using $0 . \overline{72} \notin[0.75,1 . \overline{33}]$ exactly as above, the tame-wild principle fails also for $\left(M_{11}, \phi_{11}, \phi_{12 b}\right)$ and $\left(M_{10}, \phi_{10}, \phi_{12 b}\right)$. Here the transitive permutation groups in question are respectively $(11 \mathrm{~T} 6,12 \mathrm{~T} 272)$ and (10T31, 12T181). The analog of Figure 5 for $M_{10}$ and $M_{11}$ has the same tame cone, but more dots. For $M_{12}$ there are many more dots, and a symmetry appears with the cone doubling so that its bounding lines have slope $\frac{3}{4}$ and $\frac{4}{3}$ rather than 1 and $\frac{4}{3}$.

Moving further leftward to $M_{9}$ and $Q_{8}$ relates our current discussion to our earlier counterexamples. For $M_{9}$, the transitive groups are (9T14, 12T47). However now $\hat{Q}_{8}$ is not contained in $M_{9}$ and so we do not have counterexamples over $\mathbb{Q}$. However the counterexample for $\left(M_{9}, \phi_{12 b}, \phi_{72}\right)$ over $\mathbb{Q}(\sqrt{-3})$ from Section 7D also gives a counterexample for $\left(M_{9}, \phi_{9}, \phi_{12 b}\right)$, as always because the projectivized wild Artin conductor $0 . \overline{72}$ is not in the tame hull $[0.75,1 . \overline{33}]$. Finally for $\hat{Q}_{8}$ itself we recover (8-3), now interpreted as an intransitive counterexample for $\left(Q_{8}, \phi_{8}, \phi_{8}+\phi_{4}\right)$ over $\mathbb{Q}(\sqrt{-3})$.

The extended groups $M_{9} .2$ and $M_{10} .2$ corresponding to the pairs $(9 \mathrm{~T} 19,12 \mathrm{~T} 84)$ and $(10 \mathrm{~T} 35,12 \mathrm{~T} 220)$ are natural candidates to support examples over $\mathbb{Q}$ because they contain $\hat{Q}_{8}$. However they have orbit partitions 921 and (10)2 as subgroups of $M_{12}$. Computation in the column headed by $8 \mathrm{~B}$ then has to be adjusted, with the 2 in $821^{2}$ removed. The conductor ratio is then $\frac{7}{10}$ rather than $\frac{8}{10}$ and in fact the inertial method above works to prove the tame-wild principle for $\left(M_{9} .2, \phi_{9}, \phi_{12 b}\right)$ and $\left(M_{10} .2, \phi_{10}, \phi_{12 b}\right)$. This phenomenon illustrates the fundamental difficulty in 
promoting local nontransitive counterexamples to global transitive ones with a larger group. While wild Artin conductor ratios, here $0 . \overline{72}$ stay the same, tame hulls increase, here from $[0.75,1]$ for $\hat{Q}_{8}$ itself to $\left[\frac{7}{10}, \frac{7}{6}\right]=[0.70,1.1 \overline{6}]$ for $M_{10} .2$.

There are other good candidates for global Galois groups. The 2-Sylow subgroup $P$ of $M_{12}$ of order $2^{6}$ is not good for us, because neither the given orbit decomposition nor its twin is transitive, both having orbit partition 84 . However an overgroup $T$ of order $2^{6} \cdot 3=192$ is good, with $\left(\phi_{12 a}, \phi_{12 b}\right)$ remaining a fraternal pair of type (12T112, 12T112). Our computations have shown that the tame-wild principle fails for $\left(12 \mathrm{~T} 112, \phi_{12 a}, \phi_{12 b}\right)$.

Number fields. Constructing number fields with nonsolvable Galois groups and prescribed ramification remains a difficult problem despite the increasing attention it has been receiving recently. Just as we have not found $M_{12}$ fields with the appropriate quaternionic ramification, we have also not found $M_{11}$ or $M_{10}$ fields.

In contrast, it is relatively easy to build solvable fields step by step, and we have found many explicit pairs $\left(K_{12 a}, K_{12 b}\right) / \mathbb{Q}$ providing counterexamples to the tame-wild principle for $\left(12 \mathrm{~T} 112, \phi_{12 a}, \phi_{12 b}\right)$. One such, with tame ramification at the prime number $q=277$, is

$$
\begin{aligned}
& f_{12 a}(x)=x^{12}+223 x^{10}+14856 x^{8}+1784 q x^{6}+38160 q x^{4}+1712 q^{2} x^{2}+9216 q^{2}, \\
& f_{12 b}(x)=x^{12}+202 x^{8}+49 q x^{4}+4 q^{2} .
\end{aligned}
$$

The discriminants are $D_{12 a}=2^{16} 277^{8}$ and $D_{12 b}=2^{22} 277^{6}$, with the tame prime 277 having ramification partitions $\mu_{12 a}=4^{2} 2^{2}$ and $\mu_{12 b}=4^{2} 1^{4}$.

By design, $\mathfrak{D}_{K_{12 b}}^{0.75} \nmid \mathfrak{D}_{K_{12 a}}$. However the tame ramification at 277 completely overwhelms the wild ramification at 2 in terms of magnitudes, and easily $\mid \mathfrak{D}_{K_{12 b}} \cdot{ }^{75} \leq$ $\left|\mathfrak{D}_{K_{12 a}}\right|$. Indeed $\left|\mathfrak{D}_{K_{12 b}}\right|^{1.15} \approx\left|\mathfrak{D}_{K_{12 a}}\right|$. To improve upon the counterexample $\left(f_{12 a}, f_{12 b}\right)$, one would like examples with $D_{12 a}=2^{16}\left(p_{1} \ldots p_{k}\right)^{6}$ and $D_{12 b}=$ $2^{22}\left(p_{1} \ldots p_{k}\right)^{8}$ so that (8-2) is contradicted as well. However no such counterexamples exist with $G=12 \mathrm{~T} 112$, as the subgroup $Q_{8}$ together with all elements of type $4^{2} 1^{4}$ generate an index two subgroup of type $12 \mathrm{~T} 63$ and this subgroup does not contain $\hat{Q}_{8}$. Partially summarizing:

Proposition 8.4. The tame-wild principle for $\left(G, \phi_{12 a}, \phi_{12 b}\right)$ fails for the groups $G=M_{12}, M_{11}, M_{10}$ and $12 \mathrm{~T} 112$. For $G=12 \mathrm{~T} 112$ the pair of number fields $\left(K_{12 a}, K_{12 b}\right)$ contradicts the divisibility statement (8-1), but no pair with $G=$ $12 \mathrm{~T} 112$ contradicts the numerical statement (8-2).

The group-theoretic argument for $12 \mathrm{~T} 112$ does not apply to the three $M_{n}$ and we expect that there exist pairs $\left(K_{12 a}, K_{12 b}\right)$ for them contradicting not only (8-1) but also (8-2). In general, a closer analysis of the exact range of applicability of the tame-wild principle would be interesting. 


\section{Acknowledgments}

We thank Nigel Boston and Griff Elder for helpful conversations.

\section{References}

[Amano 1971] S. Amano, "Eisenstein equations of degree $p$ in a p-adic field", J. Fac. Sci. Univ. Tokyo Sect. IA Math. 18 (1971), 1-21. MR 46 \#7201 Zbl 0231.12019

[Cameron et al. 2002] P. J. Cameron, M. Giudici, G. A. Jones, W. M. Kantor, M. H. Klin, D. Marušič, and L. A. Nowitz, "Transitive permutation groups without semiregular subgroups", J. London Math. Soc. (2) 66:2 (2002), 325-333. MR 2003f:20001 Zbl 1015.20001

[Conway et al. 1985] J. H. Conway, R. T. Curtis, S. P. Norton, R. A. Parker, and R. A. Wilson, Atlas of finite groups, Oxford University Press, Eynsham, 1985. MR 88g:20025 Zbl 0568.20001

[Conway et al. 1998] J. H. Conway, A. Hulpke, and J. McKay, "On transitive permutation groups", LMS J. Comput. Math. 1 (1998), 1-8. MR 99g:20011 Zbl 0920.20001

[Fontaine 1971] J.-M. Fontaine, "Groupes de ramification et représentations d'Artin", Ann. Sci. École Norm. Sup. (4) 4 (1971), 337-392. MR 44 \#6648 Zbl 0232.12006

[Ford and Pohst 1992] D. Ford and M. Pohst, "The totally real $A_{5}$ extension of degree 6 with minimum discriminant”, Experiment. Math. 1:3 (1992), 231-235. MR 93k:11108 Zbl 0773.11067

[Ford et al. 1998] D. Ford, M. Pohst, M. Daberkow, and N. Haddad, "The $S_{5}$ extensions of degree 6 with minimum discriminant”, Experiment. Math. 7:2 (1998), 121-124. MR 2000c:11182 Zbl 0921.11052

[Jensen et al. 2002] C. U. Jensen, A. Ledet, and N. Yui, Generic polynomials: Constructive aspects of the inverse Galois problem, Mathematical Sciences Research Institute Publications 45, Cambridge University Press, 2002. MR 2004d:12007 Zbl 1042.12001

[Jones 2013] J. W. Jones, "Minimal solvable nonic fields", LMS J. Comput. Math. 16 (2013), 130-138. MR 3065803

[Jones and Roberts 2006] J. W. Jones and D. P. Roberts, "A database of local fields", J. Symbolic Comput. 41:1 (2006), 80-97. Database at http://math.asu.edu/ jj/localfields. MR 2006k:11230 Zbl 1140.11350

[Jones and Roberts 2008] J. W. Jones and D. P. Roberts, "Octic 2-adic fields", J. Number Theory 128:6 (2008), 1410-1429. MR 2009d:11163 Zbl 1140.11056

[Jones and Roberts $\geq 2014$ ] J. W. Jones and D. P. Roberts, "A database of number fields", submitted. Database at http://hobbes.la.asu.edu/NFDB.

[Jones and Wallington 2012] J. W. Jones and R. Wallington, "Number fields with solvable Galois groups and small Galois root discriminants", Math. Comp. 81:277 (2012), 555-567. MR 2012e:11182 Zbl 06006889

[Kontorovitch 1939] P. Kontorovitch, "Sur la représentation d'un groupe fini sous la forme d'une somme directe de sous-groupes, I", Rec. Math. [Mat. Sbornik] N.S. 5 (47):2 (1939), 289-296. In Russian. MR 2,3e Zbl 0022.31304

[Kontorovitch 1940] P. Kontorovitch, "Sur la représentation d'un groupe fini sous la forme d'une somme directe de sous-groupes, II", Rec. Math. [Mat. Sbornik] N.S. 7 (49):1 (1940), 27-33. In Russian. MR 2,4a Zbl 0023.01205

[LMFDB 2013] The LMFDB Collaboration, " $L$-function and modular forms database", 2013, Available at http://www.lmfdb.org/. 
[Malle 2000] G. Malle, "Multi-parameter polynomials with given Galois group: Algorithmic methods in Galois theory", J. Symbolic Comput. 30:6 (2000), 717-731. MR 2002a:12007 Zbl 0967.12005

[Maus 1965] E. Maus, Existenz p-adischer Zahlkörper zu vorgegebenem Verzweigungsverhalten, Ph.D. thesis, Hamburg, 1965.

[Serre 1979] J.-P. Serre, Local fields, Graduate Texts in Mathematics 67, Springer, New York, 1979. MR 82e:12016 Zbl 0423.12016

[Suzuki 1950] M. Suzuki, "On the finite group with a complete partition”, J. Math. Soc. Japan 2 (1950), 165-185. MR 13,907b Zbl 0040.00703

Communicated by John Henry Coates

Received 2012-12-13 Revised 2013-09-11 Accepted 2013-10-21

jj@asu.edu

School of Mathematical and Statistical Sciences, Arizona State University, P.O. Box 871804, Tempe, AZ 85287-1804, United States

roberts@morris.umn.edu

Division of Science and Mathematics, University of Minnesota - Morris, Morris, MN 56267, United States 


\section{Algebra \& Number Theory}

msp.org/ant

\section{EDITORS}

MANAGING EDITOR

Bjorn Poonen

Massachusetts Institute of Technology

Cambridge, USA

\author{
EDITORIAL BOARD CHAIR \\ David Eisenbud \\ University of California \\ Berkeley, USA
}

\section{BOARD OF EDITORS}

Georgia Benkart

Dave Benson

Richard E. Borcherds

John H. Coates

J-L. Colliot-Thélène

Brian D. Conrad

Hélène Esnault

Hubert Flenner

Edward Frenkel

Andrew Granville

Joseph Gubeladze

Roger Heath-Brown

Ehud Hrushovski

Craig Huneke

Mikhail Kapranov

Yujiro Kawamata

János Kollár

Yuri Manin

Barry Mazur

Philippe Michel
University of Wisconsin, Madison, USA

University of Aberdeen, Scotland

University of California, Berkeley, USA

University of Cambridge, UK

CNRS, Université Paris-Sud, France

University of Michigan, USA

Freie Universität Berlin, Germany

Ruhr-Universität, Germany

University of California, Berkeley, USA

Université de Montréal, Canada

San Francisco State University, USA

Oxford University, UK

Hebrew University, Israel

University of Virginia, USA

Yale University, USA

University of Tokyo, Japan

Princeton University, USA

Northwestern University, USA

Harvard University, USA

École Polytechnique Fédérale de Lausanne
Susan Montgomery

Shigefumi Mori

Raman Parimala

Jonathan Pila

Victor Reiner

Karl Rubin

Peter Sarnak

Joseph H. Silverman

Michael Singer

Vasudevan Srinivas

J. Toby Stafford

Bernd Sturmfels

Richard Taylor

Ravi Vakil

Michel van den Bergh

Marie-France Vignéras

Kei-Ichi Watanabe

Efim Zelmanov

Shou-Wu Zhang
University of Southern California, USA

RIMS, Kyoto University, Japan

Emory University, USA

University of Oxford, UK

University of Minnesota, USA

University of California, Irvine, USA

Princeton University, USA

Brown University, USA

North Carolina State University, USA

Tata Inst. of Fund. Research, India

University of Michigan, USA

University of California, Berkeley, USA

Harvard University, USA

Stanford University, USA

Hasselt University, Belgium

Université Paris VII, France

Nihon University, Japan

University of California, San Diego, USA

Princeton University, USA

PRODUCTION

production@msp.org

Silvio Levy, Scientific Editor

See inside back cover or msp.org/ant for submission instructions.

The subscription price for 2014 is US $\$ 225 /$ year for the electronic version, and $\$ 400 /$ year $(+\$ 55$, if shipping outside the US) for print and electronic. Subscriptions, requests for back issues and changes of subscribers address should be sent to MSP.

Algebra \& Number Theory (ISSN 1944-7833 electronic, 1937-0652 printed) at Mathematical Sciences Publishers, 798 Evans Hall \#3840, c/o University of California, Berkeley, CA 94720-3840 is published continuously online. Periodical rate postage paid at Berkeley, CA 94704, and additional mailing offices.

ANT peer review and production are managed by EditFLOW ${ }^{\circledR}$ from Mathematical Sciences Publishers.

\section{PUBLISHED BY}

- mathematical sciences publishers

nonprofit scientific publishing

http://msp.org/

(C) 2014 Mathematical Sciences Publishers 


\section{Algebra \& Number Theory}

Volume $8 \quad$ No. $3 \quad 2014$

Derived invariants of irregular varieties and Hochschild homology

LUIGI LOMBARDI

Sato-Tate distributions of twists of $y^{2}=x^{5}-x$ and $y^{2}=x^{6}+1$

FRANCESC Fité and ANDREW V. SUTHERLAND

The algebraic dynamics of generic endomorphisms of $\mathbb{P}^{n}$

NAJMUDDIN FAKHRUDDIN

The tame-wild principle for discriminant relations for number fields

JOHN W. JONES and DAVID P. ROBERTS

Linear forms in logarithms and integral points on higher-dimensional varieties

AARON LEVIN

Lefschetz theorem for abelian fundamental group with modulus

689

MORITZ KERZ and ShuJi SAITO

Localization of spherical varieties

703

FRIEDRICH KNOP

Lefschetz operator and local Langlands modulo $\ell$ : the limit case

JEAN-FRANÇOIS DAT

Splitting tower and degree of tt-rings

PAUL BALMER 